\title{
WestVirginiaUniversity
}

THE RESEARCH REPOSITORY @ WVU

Graduate Theses, Dissertations, and Problem Reports

2005

\section{Robust pre-processing techniques for non-ideal iris images}

\author{
Purva M. Barve \\ West Virginia University
}

Follow this and additional works at: https://researchrepository.wvu.edu/etd

\section{Recommended Citation}

Barve, Purva M., "Robust pre-processing techniques for non-ideal iris images" (2005). Graduate Theses, Dissertations, and Problem Reports. 1656.

https://researchrepository.wvu.edu/etd/1656

This Thesis is protected by copyright and/or related rights. It has been brought to you by the The Research Repository @ WVU with permission from the rights-holder(s). You are free to use this Thesis in any way that is permitted by the copyright and related rights legislation that applies to your use. For other uses you must obtain permission from the rights-holder(s) directly, unless additional rights are indicated by a Creative Commons license in the record and/ or on the work itself. This Thesis has been accepted for inclusion in WVU Graduate Theses, Dissertations, and Problem Reports collection by an authorized administrator of The Research Repository @ WVU. For more information, please contact researchrepository@mail.wvu.edu. 


\title{
ROBUST PRE-PROCESSING TECHNIQUES FOR NON-IDEAL IRIS IMAGES
}

\author{
By \\ Purva M Barve \\ Thesis submitted to the College of Engineering and Mineral Resources at \\ West Virginia University \\ In partial fulfillment of the requirements for the degree of
}

\section{MASTER OF SCIENCE \\ In \\ ELECTRICAL ENGINEERING}

\author{
Approved by \\ Dr. Xin Li (Chair) \\ Dr. Arun Ross \\ Dr. Natalia Schmid \\ Lane Department of Computer Science and Electrical Engineering \\ West Virginia University \\ Morgantown, West Virginia \\ 2005 \\ Keywords: \\ Iris Recognition, Non-ideal, Pre-processing, Robust Segmentation
}




\title{
ABSTRACT \\ ROBUST PREPROCESSING TECHNIQUES FOR NON-IDEAL IRIS IMAGES
}

\author{
By \\ PURVA MADHAV BARVE
}

The human iris has been demonstrated to be a very accurate, non-invasive and easy-to-use biometric for personal identification. Most of the current state-of-the-art iris recognition systems require the iris acquisition to be ideal. A lot of constraints are hence put on the user and the acquisition process.

Our aim in this research is to relax these conditions and to develop a preprocessing algorithm, which can be used in conjunction with any matching algorithm to handle the so-called non-ideal iris images. In this thesis we present a few robust techniques to process the non-ideal iris images so as to give a segmented iris image to the matching algorithm. The motivation behind this work is to reduce the false reject rates of the current recognition systems and to reduce the intra-class variability. A new technique for estimating and compensating the angle in non-frontal iris images is presented. We have also developed a novel segmentation algorithm, which uses an ellipse-fitting approach for localizing the pupil. A fast and simple limbus boundary segmentation algorithm is also presented. 
(C) Copyright 2005 Purva M Barve All Rights Reserved 


\section{DEDICATED TO MY PARENTS}




\section{ACKNOWLEDGEMENTS}

I have been privileged to have Dr. Xin Li as my advisor. His quest for excellence, attention to detail and ingenious ideas has always encouraged me to give my best time and again. I wish to thank him whole- heartedly for making my Masters' experience a memorable one. It was my wish to work with him ever since I decided to go for a Masters Degree and I am very glad he gave me the opportunity.

I am very grateful to Dr. Arun Ross and Dr. Natalia Schmid for their constant encouragement and support throughout the course of the program.

I have been fortunate enough to be surrounded by a wonderful circle of friends who were with me even through my hard times. They really made life in a different country very easy for me. I would like to thank my colleagues for engaging in technical discussions and giving insightful comments. May you succeed in all your endeavors.

I would not have come this far without the ever-lasting support of my mother and my sister Prajakta. I am very grateful to my mother for being strong and encouraging me to fulfill my father's wishes. He would have been very happy to see me at this juncture in my life.

Last but not the least I would like to thank my fiancé, Anirudha for understanding me, standing by me and sharing my joys and frustrations.

To all of you I dedicate this work. 


\section{TABLE OF CONTENTS}

List of Figures

List of Tables

ix

\section{Introduction}

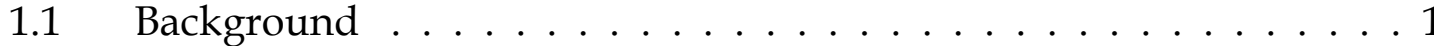

1.1.1 Iris as a Biometric . . . . . . . . . . . . . 2

1.1.2 Interesting facts about the Iris . . . . . . . . . . . . . . . 4

1.1.3 Previous Work related to Iris Recognition . . . . . . . . . . . . 5

1.1.4 "Non-Ideal" Iris Images. . . . . . . . . . . . . . . . . . . . . 9

1.2 Problem Statement . . . . . . . . . . . . . . . . . . . . . . . . . . . 13

1.3 Contributions of this thesis . . . . . . . . . . . . . . . 16

1.4 Thesis Organization . . . . . . . . . . . . . . . 17

\section{Robust Segmentation}

2.1 Motivation . . . . . . . . . . . . . . . . . . . . . . . . . 19

2.2 Pupil Detection and Localization . . . . . . . . . . . . . . 20

2.3 Limbus Segmentation. . . . . . . . . . . . . . . . . . . . . 25

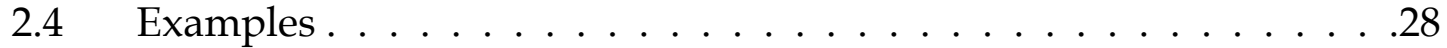

\section{Dealing with Off-angle Iris Images}

3.1 Problem Description . . . . . . . . . . . . . . . . . . . . 31

3.2 Proposed Solution. . . . . . . . . . . . . . . . . . . . . . .33

3.2 .1 Angle Estimation . . . . . . . . . . . . . . . . . . . . . .33

3.2.2 Angle Compensation . . . . . . . . . . . . . . . . . . 36

3.3 Examples . . . . . . . . . . . . . . . . . . 37

\section{Results}

$4.1 \quad$ CASIA Database. . . . . . . . . . . . . . . . . . . . 42

4.2 Eye Institute Database . . . . . . . . . . . . . . . . . . . 44

4.3 WVU Database . . . . . . . . . . . . . . . . . . . 46

$4.4 \quad$ UB Iris Database. . . . . . . . . . . . . . . . . . . . . . . . . . 47

\section{Towards Future Research}

$5.1 \quad$ Non-ideal Imaging . . . . . . . . . . . . . . . . . . . . 50

5.1 .1 Defocus/Motion Blur . . . . . . . . . . . . . . . . . .50

5.1 .2 Eyelid/Eyelash Detection. . . . . . . . . . . . . . . . . . .51

5.1 .3 Super-resolution . . . . . . . . . . . . . . . . .51

$5.2 \quad$ Iris Classification. . . . . . . . . . . . . . . . . . . . .53

5.3 Using Multiple Templates . . . . . . . . . . . . . . . . . . 57

6 Conclusions......................... . . . . .

Bibliography . . . . . . . . . . . . . . . . .61 


\section{LIST OF FIGURES}

1.1 Examples of some biometric modalities used in authentication . . . . . . 2

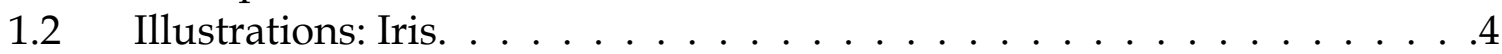

1.3 Block Diagram of Daugman's Iris Recognition System . . . . . . . . . . 6

1.4 White Spots Due to Specular Reflections. . . . . . . . . . . . . . . 10

1.5 Varying Lighting Conditions. . . . . . . . . . . . . . . . . . . 11

1.6 Varying angle of Acquisition. . . . . . . . . . . . . . . . . . . . . . . . . . . .

1.7 Blurred image due to improper focus. . . . . . . . . . . . . . . . . . . . . . . . . . .

1.8 Occlusion of iris due to eyelids/eyelashes. . . . . . . . . . . . . 12

1.9 Varying size of pupil due to contraction/dilation. . . . . . . . . . . 13

1.10 Iris Recognition System. . . . . . . . . . . . . . . . . . . . . . . . . . . . . . . . . .

1.11 Example Images from different databases. . . . . . . . . . . . . . . . 15

2.1 Illustration: Iris Segmentation . . . . . . . . . . . . . . . . . . . . . . . . .

2.2 Loss of data due to circular-based transform . . . . . . . . . . . . . . . 19

2.3 Improper segmentation due to specular reflections . . . . . . . . . . . . . . . . . . . . .

2.4 Output after binarization . . . . . . . . . . . . . . . . . . . . . . . . . . . . . . . .

2.5 Output after morphological processing. . . . . . . . . . . . . . . . . . . . . . . . . . . . . . .

2.6 Output after step 2 (White boundary- Pupil boundary) . . . . . . . . . . 24

2.7 Results of Pupil localization . . . . . . . . . . . . . . . . . . . . . . . . . . . . . . . . . . . .

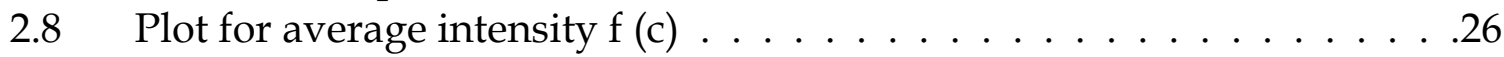

2.9 Plot for average intensity $\mathrm{f}$ (c) after non-linear diffusion . . . . . . . . . 27

2.10 Segmentation in presence of specular reflections. . . . . . . . . . . 28

2.11 Correct segmentation of non-circular pupil . . . . . . . . . . . . . . . 29

2.12 Segmentation of blurred image . . . . . . . . . . . . . . . . 29

2.13 Segmentation in presence of occlusions. . . . . . . . . . . . . 30

3.1 Images with varying angle of acquisition. . . . . . . . . . . . . . . . . . . . .

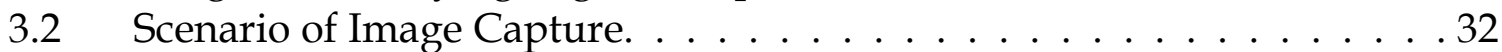

3.3 Coarse to fine approach for angle estimation. . . . . . . . . . . . . 34

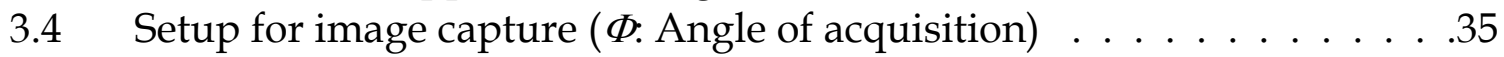

3.5 Approximation of pupil as circle in (a) Front Angle and as ellipse in (b)

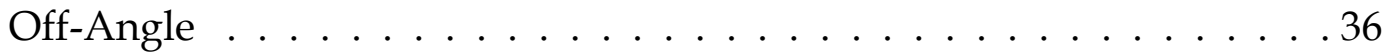

3.6 A perspective transformation with center $O$, mapping the plane $P$ to the plane Q. . . . . . . . . . . . . . . . . . . . . 37

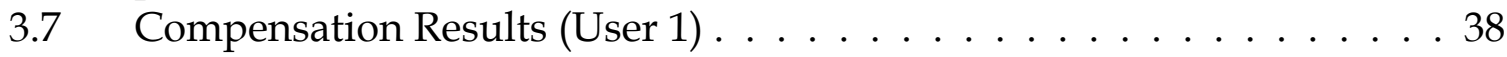

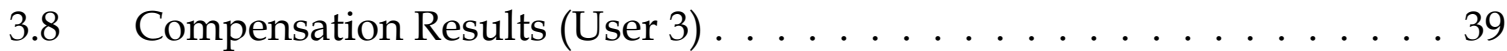

4.1 Segmentation Results for CASIA Database. . . . . . . . . . . . . . . 44

4.2 Segmentation Results for Eye Institute Database . . . . . . . . . . . . . 45

4.3 Segmentation Results for WVU Non-Ideal Database. . . . . . . . . . . . 47

4.4 Segmentation Results for UB Iris Database. . . . . . . . . . . . . . . . . . 49 
5.1 ROC Curves for CASIA Dataset using Daugman' s Algorithm. . . . . . 52

5.2 Iris Enhancement and super-resolution. . . . . . . . . . . . . 53

5.3 Stream Iris. . . . . . . . . . . . . . . . . . . . . . . . . . 54

5.4 Flower Iris. . . . . . . . . . . . . . . . . . . . . . . . . . . 55

5.5 Jewel Iris. . . . . . . . . . . . . . . . . . . . . . . . 56

5.6 Shaker Iris. . . . . . . . . . . . . . . . . . . . . . 57 


\section{LIST OF TABLES}

Table 1 Databases Used . . . . . . . . . . . . . . . . . . . . . . 15

Table 2 Scoring Mechanism (X - Don't Care) . . . . . . . . . . . . . 41

Table 3 Overall Results of proposed algorithm . . . . . . . . . . . . 41

Table 4 Scores for CASIA Database. . . . . . . . . . . . . . . . . . 43

Table 5 Scores for Eye Institute Database . . . . . . . . . . . . . . . . 45

Table 6 Scores for WVU Database . . . . . . . . . . . . . . . . . . . 46

Table $7 \quad$ Scores for UB Iris Database . . . . . . . . . . . . . . . . . 48 


\section{Chapter 1}

\section{Introduction}

\subsection{Background}

In recent years, with increase in number of security breaches and transaction frauds, secure personal identification and verification technologies have gained importance. A large number of systems thus require some kind of reliable personal authentication. The purpose of such systems is to ensure that the rendered services are being accessed by a legitimate user and not by anyone else. Most conventional systems are based on "what you possess" (e.g. An identity card) or "what you remember" (e.g. password). Biometrics allows us to establish a user's identity based on "who you are" or "what you do" [1].

Biometrics is the automatic identification (or verification) of an individual (or a claimed identity) using the physiological or behavioral characteristics of that individual. Any human physiological and/or behavioral characteristic can be used as a biometric characteristic as long as it satisfies the following requirements: uniqueness, universality, permanence, collectability, performance, acceptability and circumvention [2]. Biometric technologies are growing to be the basis of an extensive range of highly secure identification and verification solutions. 


\subsubsection{Iris as a Biometric}

As said before, biometrics makes use of certain physiological and behavioral characteristics of a person for identification or verification. These characteristics are called biometric 'modalities' or 'traits'. The physical characteristics like fingerprints, hand geometry, iris, retina, face, hand vein, facial thermograms, palmprint and behavioral characteristics like signature, voiceprint, gait, keystroke dynamics, etc. are examples of biometric modalities. Fig 1.1 illustrates some of the modalities used in biometrics.

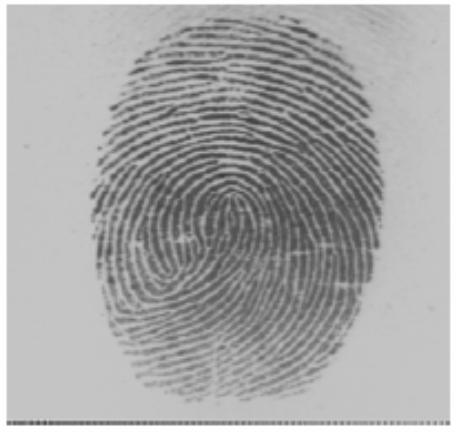

(a) Fingerprint

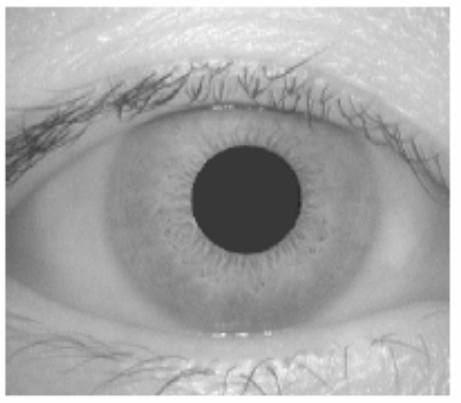

(d) Iris

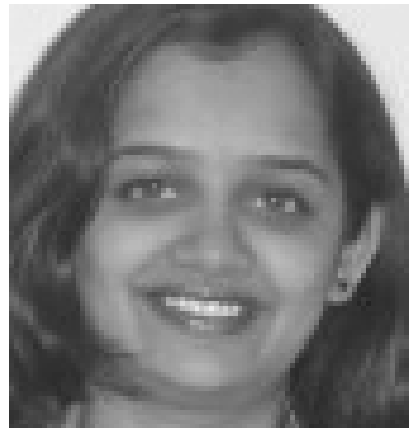

(b) Face

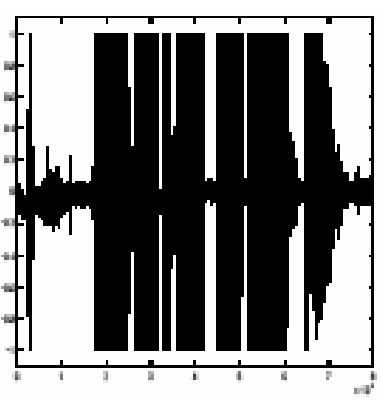

(e) Voice

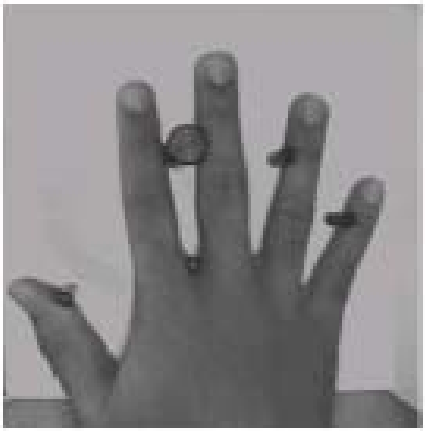

(c) Hand Geometry

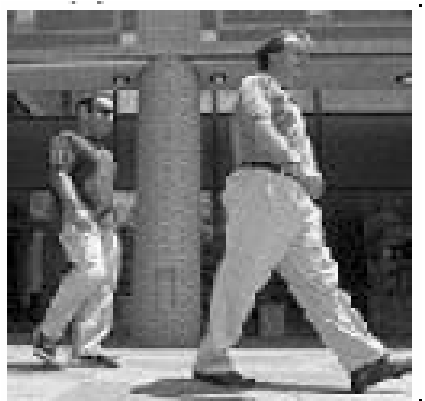

(f) Gait

Fig 1.1: Examples of some biometric modalities used in authentication

Any trait, to be used as a biometric, needs to satisfy certain conditions. Biometrics such as signature, gait, voice, face and even fingerprint, all have significant drawbacks. The problem faced by most of the behavioral traits is that they are highly likely to change over time. The uniqueness of these traits is also 
an issue. Face as a biometric has the problem because of the intra-class similarity caused due to the likeness of son with father, between siblings or twins. Fingerprints though do not have these problems, require physical contact during acquisition and are also highly susceptible to deformations and noise.

There are a number of factors that weigh heavily in favor of iris as a biometric, especially for those applications that require very large databases and real-time authentication. Let us see some of the advantages of using iris as a biometric.

- An iris pattern is absolutely unique. Not only are iris patterns of different individuals different, but even a persons left and right eye have completely unique iris patterns. It has been calculated that the chance of finding two randomly formed identical irises is on an almost astronomical order of 1 in $10^{78^{*}}$.

- Iris patterns are formed at embryonic stage and continue developing till age 1 , after which they are constant till death. The only exceptions would be accidents or surgery. This is another main advantage of iris as a biometric; almost every other biometric template changes significantly over time. This then necessitates frequent enrollment and also affects the system performance.

- It has been proved that state of the art iris recognition systems capture about 249 degrees of freedom [3] [4]. Fingerprints, facial recognition and hand geometry do not provide as many details to help in formation of the template. This is one reason why iris recognition can authenticate with confidence even when significantly less than the whole eye is visible.

- Another important factor that makes iris technology popular is the processing speed. Most iris recognition systems deliver 1-n searching of large databases in real time. This is the area in which iris is favored over fingerprint

* www.lgiris.com/iris/compares.html 
technology since fingerprint searches are challenged by database size, adding time to searches or necessitating filtering as a search acceleration technique.

- Iris capture is very non-invasive since there is no use of bright lights or lasers. Unlike some other popular biometrics, like fingerprint and hand geometry, iris authentication involves no physical contact.

A highly accurate technology such as iris-scan thus has vast appeal because the inherent argument for any biometric is increased security.

\subsubsection{Interesting Facts about The Iris}

The human iris is the annular and pigmented ring that surrounds the pupil of the eye. The iris, visible through the clear cornea, is a thin diaphragm composed mostly of connective tissue and smooth muscle fibers. It lies between the cornea and the crystalline lens.[5] (Refer Fig 1.2).

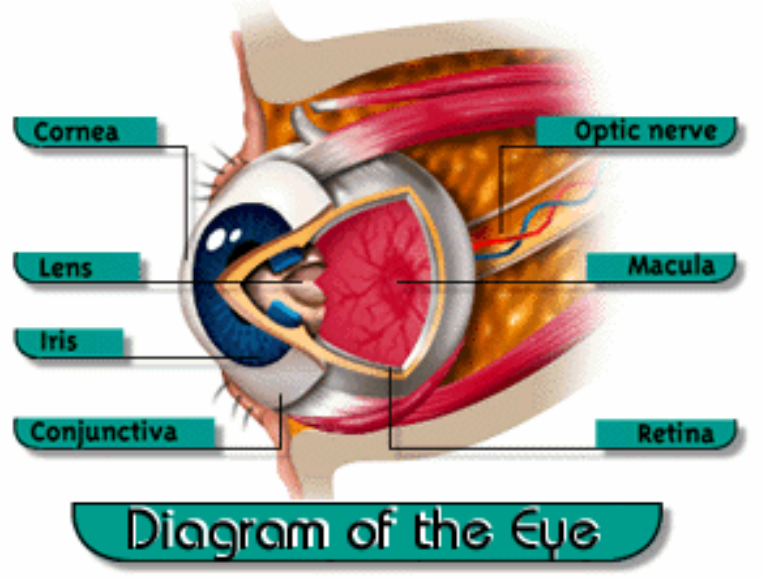

(a)

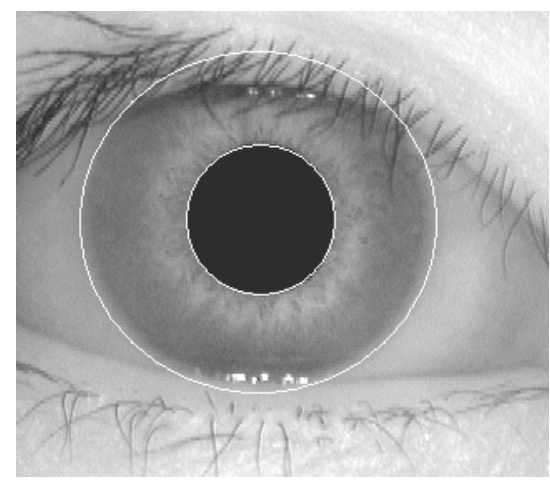

(b)

Fig 1.2: (a) Parts of Eye* (b) Iris

*http://www.2eyeballs.com/diagram.html 
The iris is composed of 3 layers, from the front to the back:

\section{Endothelium}

2. Stroma

3. Epithelium

The color of the iris, which is established genetically, is determined by the amount of pigment present in this eye structure. Contrary to popular belief, the iris does not change colors in an adult (except in the case of certain pathologies, such as pigment dispersion syndrome), even though it may be apparent, depending upon the color of clothing a person is wearing.

The iris acts like the shutter of a camera; while the pupil, which is the (normally) circular hole in the middle of the iris, is similar to the aperture of a camera. The pupil regulates the amount of light passing through to the retina, which is placed at the back of the eye. As the amount of light entering the eye diminishes (such as in the dark or at night), the iris dilator muscle (which runs radially through the iris like spokes on a wheel) pulls away from the center, causing the pupil to "dilate", allowing more light to reach the retina. When too much light is entering the eye, the iris sphincter muscle (which encircles the pupil) pulls toward the center, causing the pupil to "constrict" and allowing less light to reach the retina. Constriction of the pupil also occurs when the crystalline lens changes focus to a near distance; this reaction is known as the "near reflex."

\subsubsection{Previous Work related to Iris Recognition}

Ophthalmologists Leonard Flom and Aran Safir [10] were awarded a patent in 1987 for recognizing that human irises possess unique features that may be used to identify people. Although prototype Iris recognition systems had been proposed earlier, it was not until the early nineties that Cambridge researcher, 
John Daugman, implemented a working prototype of an automated iris recognition system [7][8][9].

Let us have a detailed look at the traditional and most popular Daugman 's system for iris recognition. Fig 1.3 summarizes the main modules of the system. The first step is to acquire the iris image. Various types of cameras can be used here; capturing images in infrared light is mostly used since it gives good quality images, with lesser noise than visible light images. The next is the image processing step where the iris is segmented from the available image. This is then converted to a simple-to-process rectangular strip using effective integrodifferential operators. Each isolated iris pattern is then demodulated to extract its phase information using quadrature 2D Gabor wavelets.
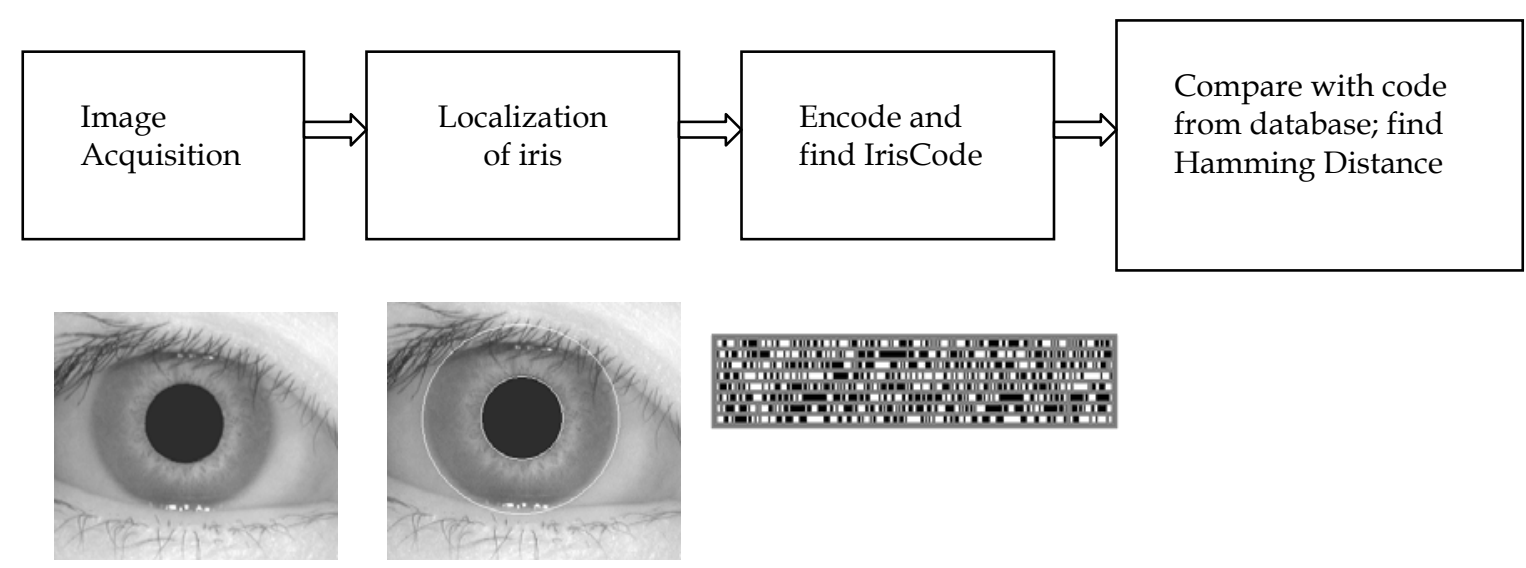

Fig 1.3: Block Diagram of Daugman's Iris Recognition System

Only phase information is used for recognizing irises because amplitude information is not very discriminating, and it depends upon extraneous factors such as imaging contrast, illumination and camera gain. The angle of each phasor is quantized to one of the four quadrants, setting two bits of phase information. This process is repeated all across the iris with many wavelet sizes, frequencies, and orientations and 2048 bits forming an IrisCode are extracted. The same number of masking bits is also computed to indicate whether any iris region is 
obscured by eyelids, contains any eyelash occlusions, specular reflections and thus should be ignored in the demodulation code as an artifact.

Then, while authentication, the incoming user's IrisCode is compared with that from the database and the Hamming Distance between them is calculated as in Equation 1.1.

$$
H D=\frac{\left\|(\operatorname{code} A \otimes \operatorname{code} B)^{\wedge} \operatorname{mas} k A \wedge \operatorname{mas} k B\right\|}{\left\|\operatorname{mask} A^{\wedge} \operatorname{mask} B\right\|}
$$

where mask $A$ and maskB denote the masking bits generated for codeA and codeB respectively, $\otimes$ is the XOR operator and I is the AND operator. The L2 norm $(\|\cdot\|)$ is used to calculate fractional HD as a measure of dissimilarity between the two IrisCodes, with the value of 0 for a perfect match. The HD is then compared with a threshold to decide whether the candidate user is a Genuine or an Imposter.

In other approaches, Wildes [11][12] makes use of the Laplacian of the Gaussian filter to extract the features in the texture part. The matching is done based on computation of the normalized correlation between the acquired and database representations. Boles, Boashash proposed a technique based on wavelet transform zero crossings [13]. The localization uses edge detection. For matching purposes, two dissimilarity functions are defined using information from the zero crossings. This is translation, rotation and scale invariant and insensitive to lighting conditions.

Ma, Tan, Wang [14] [15], presented recognition techniques using multiple Gabor filters. In the method proposed in 2000, 8-directional Gabor filters with multiple frequencies are used and the mean and variance values of these Gabor filtered images are used as features for matching. In 2004 they proposed another technique [16] based on characterizing key local variations in the iris pattern. 
This uses the 1-D dyadic wavelet transform technique. Matching is done with the help of a modified Hamming distance. They do not include the details of localization techniques used. They subsequently proposed a few improvements to their algorithm [17][18].

Noh et.al [20] proposed an iris recognition system that makes use of Independent Component Analysis (ICA) to generate optimal basis vectors, which are then used to extract efficient feature vectors representing the iris signals. Huang, Luo, Chen [21] in 2002 also used ICA. In localization, outer boundary is first detected based on a histogram. Coarse estimates were prepared using edge information and an integro-differential operator is used to improve them. The important feature of this algorithm is that it detected eyelids as well as eyelashes using Hough Transform. They estimate ICA coefficients for small windows of the iris image and generate feature vectors by quantizing them. Average Euclidean distance facilitates matching. Dorairaj et.al [25] recently proposed a global encoding technique using PCA in conjunction with ICA to give an improved performance. They also addressed some of the issues in pre-processing.

Tisse et. al [22] in 2002 used a 2D Hilbert transform to obtain instantaneous phase and/or emergent frequency information. Their localization takes into account spot reflections and uses the integro-differential operators like in Daugman 's technique. Hamming distance is used for matching.

L. Masek [23] in his Master's Dissertation proposed an 'open-source' iris recognition system in order to verify both the uniqueness of the human iris and also its performance as a biometric. The iris recognition system consists of an automatic segmentation system that is based on the Hough transform, and is able to localize the circular iris and pupil region, occluding eyelids and eyelashes, and reflections. Hamming distance was used as a matching measure. 
Thus we can see here that though some of the algorithms do take into account the noise in iris images, none of them handle all of the factors effectively. Also a point to be noted is that all of them make the assumption that the pupil is circular. We aim to solve these problems through this work.

\subsubsection{Non-Ideal Iris Images}

We can thus say that any Iris Recognition system consists of three main parts: Image Acquisition, Pre-processing and Encoding/Matching. Most of the current literature focuses on operating with iris images, which have been obtained by full co-operation from the user. These systems thus make an assumption of an ideal image acquisition system.

This is however, not always the case in practice. Let us consider examples of some of the practical applications of iris recognition systems. Frequently, in border control applications and even at airports, iris recognition is a part of a larger system, where the user's face is imaged and the eyes are localized from these facial images. In these cases, it is not possible to always ensure that the distance between the eye and the camera is as small as is required by general recognition systems. Hence, we may not be able to obtain iris images of required resolutions. In border crossing applications, the large number of users and limited time available might introduce blurring in the captured images. Thus, the issue is that in our busy world we are facing more and more situations of iris imaging where we cannot rely on high quality iris acquisition.

Most recognition systems require that the user look directly at the camera. If the angle of acquisition of the image changes even a little, it might lead to a legitimate user being rejected. Such a system cannot be applied in scenarios where the users cannot be expected to fully co-operate; e.g. hospitals or schools. 
Also, occlusion might be caused because of eyelids/ eyelashes in such scenarios.

Thus, considering that real-world images are non-ideal, the area of preprocessing gains a lot of importance. The quality of the captured image will depend on the constraints put on the image acquisition. As we go on relaxing these constraints, the quality of the captured image degrades and preprocessing it to obtain a good quality image becomes necessary. This is because the quality of segmentation of the iris from the image, which is the most important part of pre-processing, directly affects the recognition performance of the system.

Having said this, let us take a closer look at what characterizes the "non-ideality" of iris images along with some image examples.

There are a number of factors that play a role here:

- Specular Reflections:

Depending on the type of camera and lighting conditions (visible/ infrared etc.) there can be reflections at different positions and of varying amounts. The white spots caused by these reflections lead to loss of information and also pose problems for segmentation. Fig 1.4 shows some images affected by specular reflections.
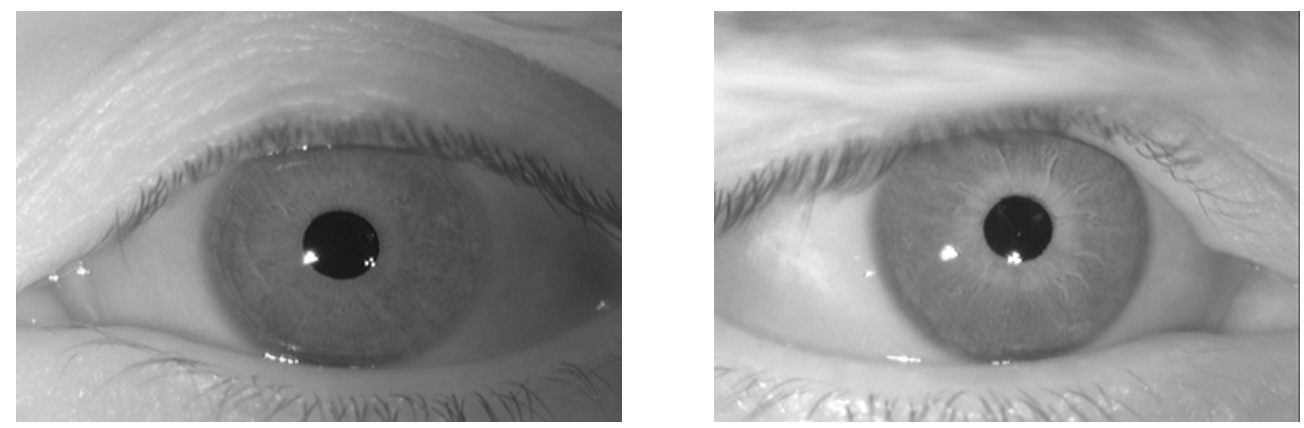

Fig 1.4: White Spots Due to Specular Reflections 
- Lighting Conditions:

Varying amount of light is also a major factor, which contributes to the nonideality of the captured images. As seen in Fig 1.5, the images might sometimes be dark because of shadows or too bright. This makes it very difficult to process the iris images.
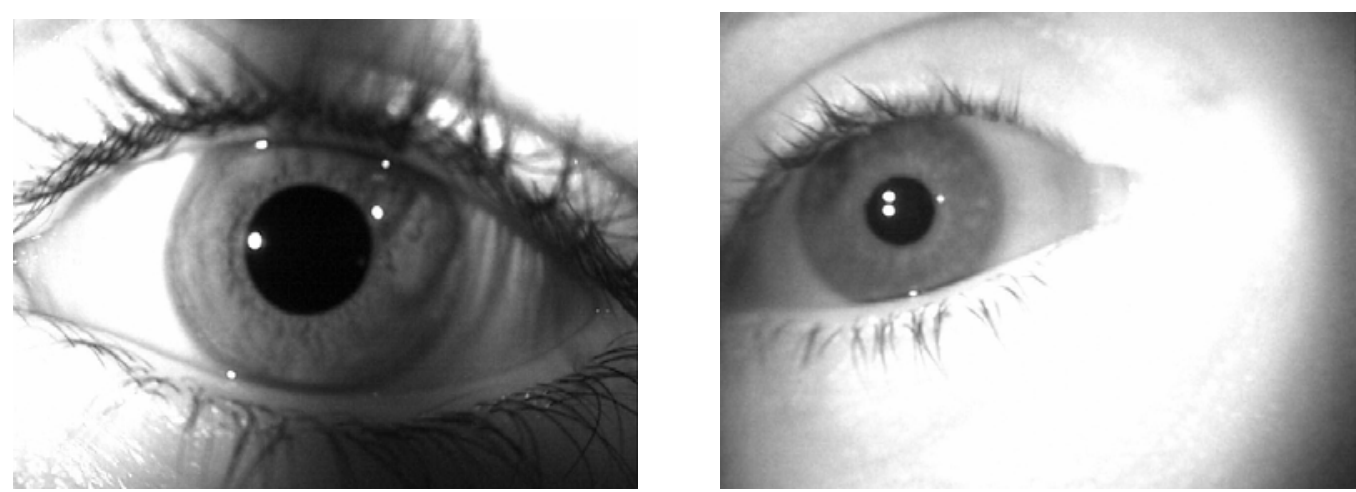

Fig 1.5: Varying Lighting Conditions

- Angle of Acquisition:

Another extremely important and so far ignored issue is the varying angle of acquisition of the iris image. Depending on the angle at which the image has been captured, a slightly "different" image is captured. Matching such images, which are not captured from same angle, leads to high intra-class distances. There hence has to be some pre-processing of these images. Fig 1.6 shows two images of the same eye captured from different angles.
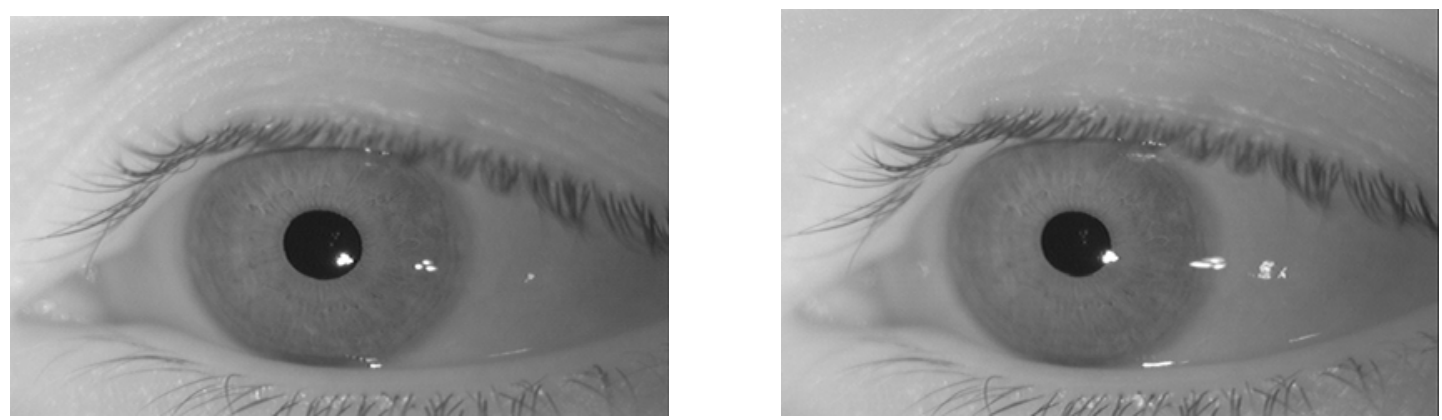

Fig 1.6: Varying angle of Acquisition 
- Defocus Blur:

Sometimes the non-ideality is due to the fact that the focusing has not been done properly. This leads to blurring in the images. This is also a very important aspect that we have to contend with before encoding the iris image. Fig 1.7 illustrates the defocus blur.

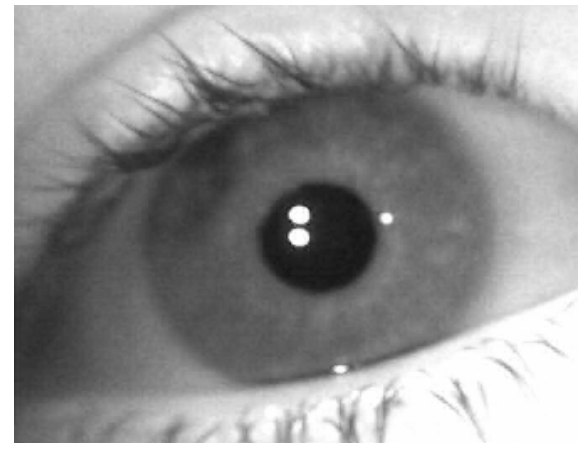

Fig 1.7: Blurred image due to improper focus

- Motion Blur:

Similar to defocus blur, motion on the part of the user or the camera might introduce blur. This is also another cause of non-ideal iris images.

- Eyelid/Eyelash Occlusions:

This is the most commonly observed degradation in the captured iris images. The eyelashes could obstruct some part of the iris if anyone has very long eyelashes; or the eyelids if the user happens to close the eye a bit more than actually required for good image capture. Fig 1.8 illustrates the occlusion caused by eyelids/eyelashes in iris images.
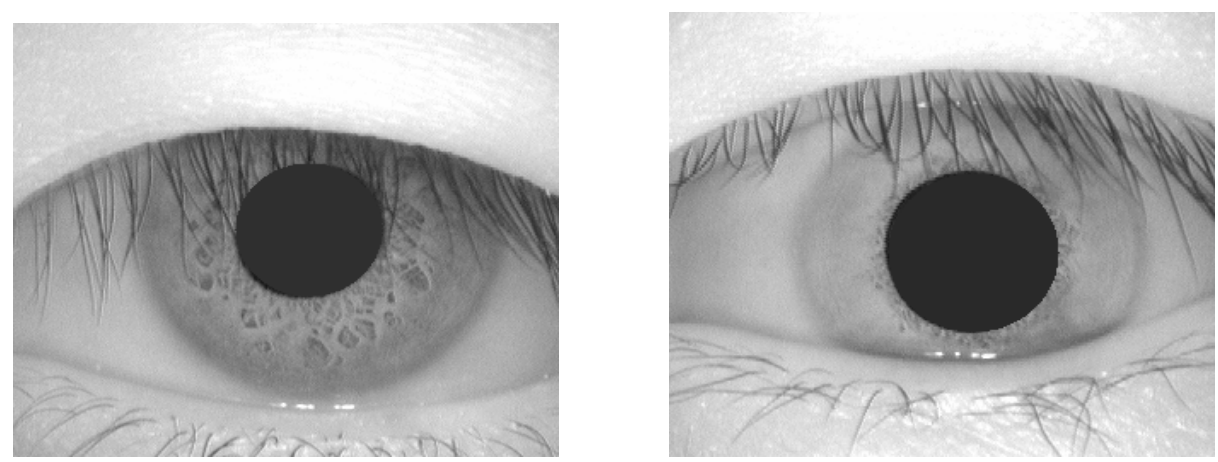

Fig 1.8: Occlusion of iris due to eyelids/eyelashes 
- Pupil Contraction/Dilation:

The varying lighting conditions could also have some effect on the size of the pupil of the eye, this in turn bringing about change in the width of the iris pattern. When bright light is shone on the eye, it automatically constricts. This is the pupillary reflex, which protects the retina from bright light. When the sphincter muscle within the iris contracts, it reduces the size of the pupil. The human pupil dilates if the person sees something (or someone) of interest. Dilation occurs when signals from sympathetic fibers cause the iris dilator muscles (dilator pupilae) to contract. As shown in Fig 1.9, the size of the pupil can thus vary to a large extent. This poses a problem to both the localization and the encoding parts of the iris recognition system.
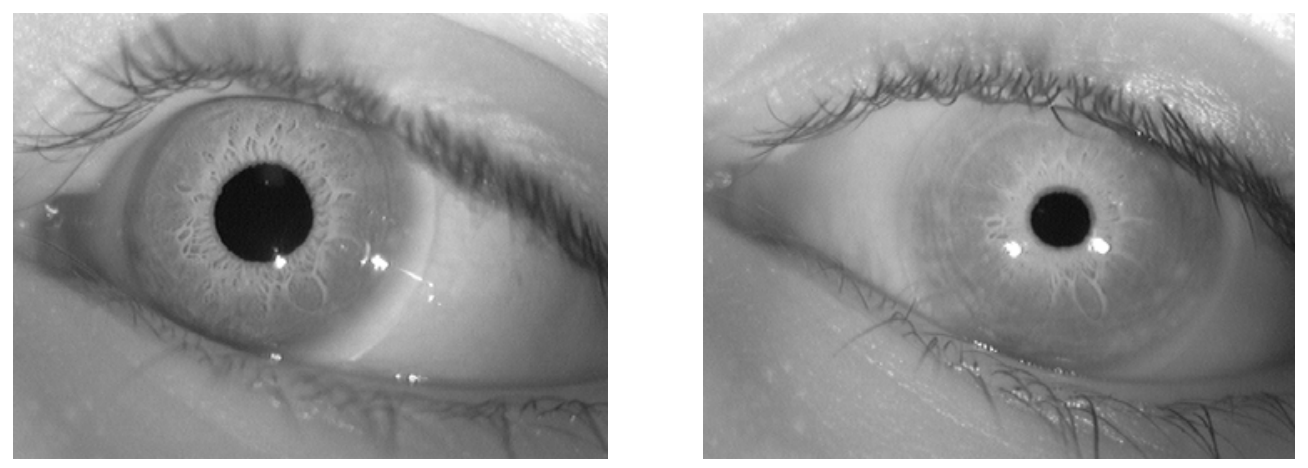

Fig 1.9 Varying size of pupil due to contraction/dilation

\subsection{Problem Statement}

The previous section detailed the various factors that could lead to "non-ideal" images. Our aim in this work is to develop various image processing tools to address these issues and give a good quality segmented image to the encoding part of the iris recognition system. 


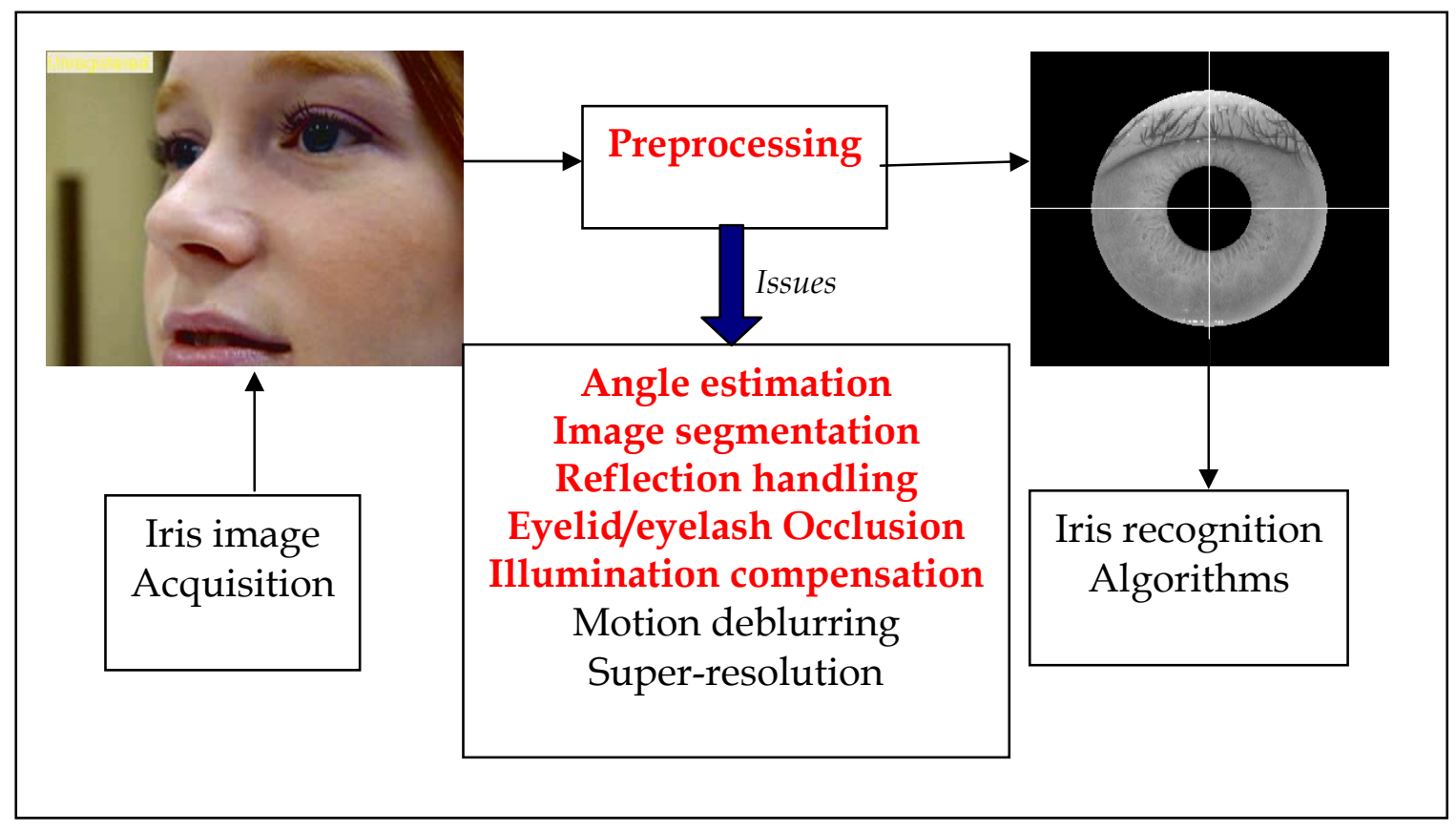

Fig 1.10: Iris Recognition System

Fig 1.10 summarizes acquisition and preprocessing steps. We have concentrated on solving the problems of robust segmentation and varying angle of acquisition. The aim in robust segmentation is to create a universal algorithm, which effectively localizes the iris in varied qualities of iris images. The robustness refers to the ability of the algorithm to handle various issues as shown in the figure. The ones highlighted in the figure are those we have successfully handled in our proposed algorithm.

To accommodate the variations in the quality of real- world images, we have used four different databases of iris images. Table 1.1 summarizes the available data. Fig 1.11 shows example images from the databases. Off-angle images were available only for the Eye Institute Database. The images in the UB Iris Database are captured in visible light whereas other in infrared light. More about the databases and the results we obtained for the same has been presented in Chapter 4. 


\begin{tabular}{|l|c|c|c|}
\hline Databases & Number of users & Images Available & Off-Angle Images \\
\hline CASIA Database & 108 & 6 per user & No \\
WVU Database & Not labeled & 20 & No \\
$\begin{array}{l}\text { Eye Institute } \\
\text { Database }\end{array}$ & 100 & 8 per user & Yes; 2 per user \\
UB Iris Database & 240 & 5 per user & No \\
\hline
\end{tabular}

Table 1: Databases Used

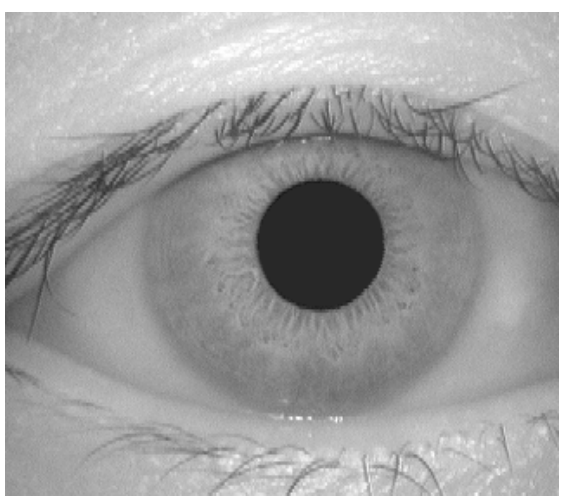

(a) CASIA Database

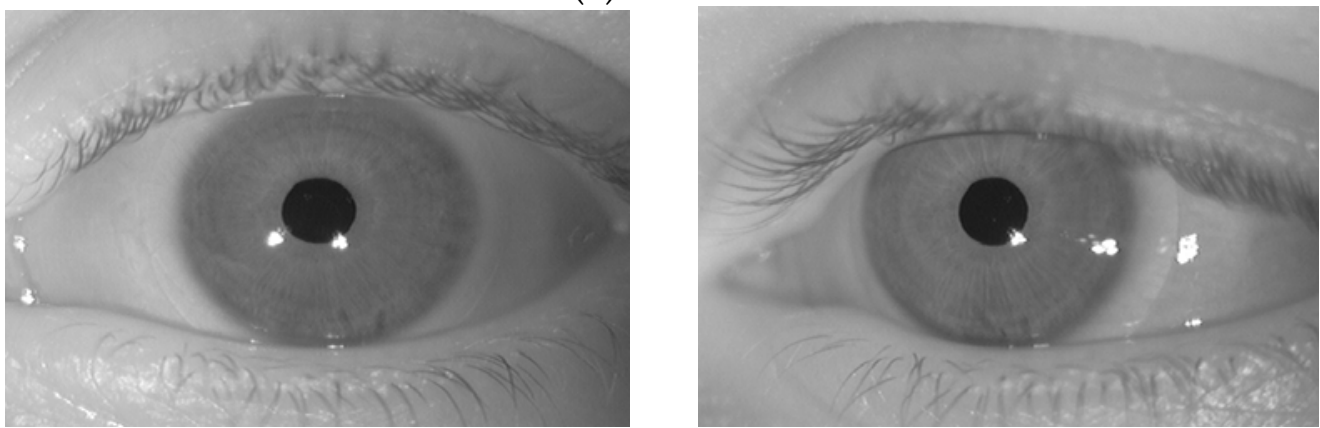

(b) Eye Institute Database

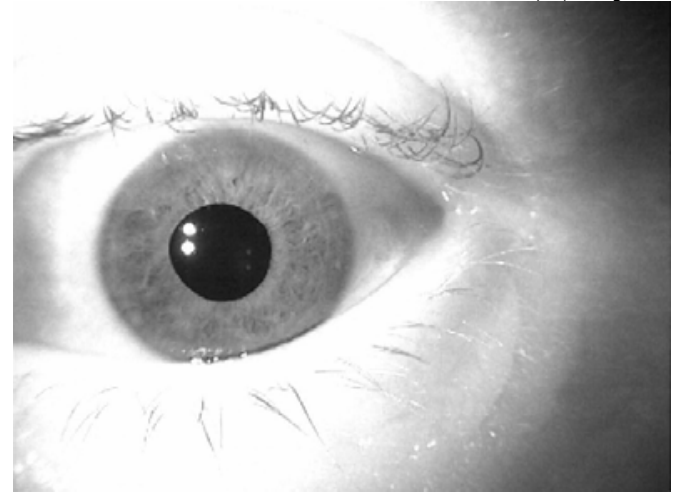

(c) WVU Database

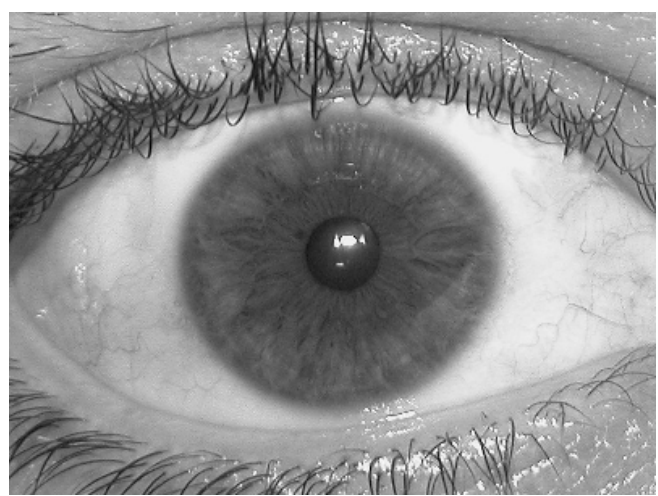

(d) UB Iris Database

Fig 1.11: Example Images from different databases 


\subsection{Contributions of this thesis}

The problem of pre-processing has not been addressed adequately in the research to date on iris recognition. Most of the research makes the assumption of good quality iris images. In our work we have successfully handled the important issues associated with non-ideal images.

The first contribution is the novel approach of localization of the iris pattern from the entire image. The algorithm compensates for specular reflections and variations in the size of the pupil. Our approach effectively determines the areas affected by reflections and masks them in further processing. Also a very important aspect of this part of our work is that this algorithm can segment the iris successfully even if the pupil is not exactly circular. The segmentation of the limbic boundary (outer boundary) of the iris takes into account the occlusions due to eyelids/eyelashes. We have included a few morphological image processing tools in our algorithm, that, to a certain extent, assist in dealing with the problems caused by varying lighting conditions.

Secondly, this work also deals with the problem of varying angle of acquisition. Our novel approach of segmentation has another advantage; it plays an important role in estimating the angle. The scenario considered here is such that the gaze direction and scene depth remain constant. Hence the deformation in the image due to change in capture angle is only along the horizontal direction. Having made these assumptions, we have successfully estimated the angle and compensated the image for that angle using geometric transformations. 


\subsection{Thesis Organization}

In Chapter 2, we describe the approach taken for robust segmentation of the iris images along with a few examples. Chapter 3 deals with the problem of varying angle of acquisition. The techniques for the estimation and compensation of the angle are described. Chapter 4 details the experimental results and overall performance of the algorithm. Chapter 5 outlines the approaches that could be taken for effectively addressing the issues with non-ideal images not dealt with by our algorithm. We also give some of our preliminary results regarding the same. It includes scope for further research. Chapter 6 provides the conclusions. 


\section{Chapter 2}

\section{Robust Segmentation}

The first and most important part of pre-processing is the effective segmentation of the iris pattern. This segmentation presents a challenge due to the non-ideality of the available iris images. The localization of the iris consists of two parts:

- Localization of the pupil and hence the inner boundary or pupillary boundary of the iris, and

- Separation of the iris pattern from the sclera; i.e. marking the outer boundary or the limbus boundary of the iris.

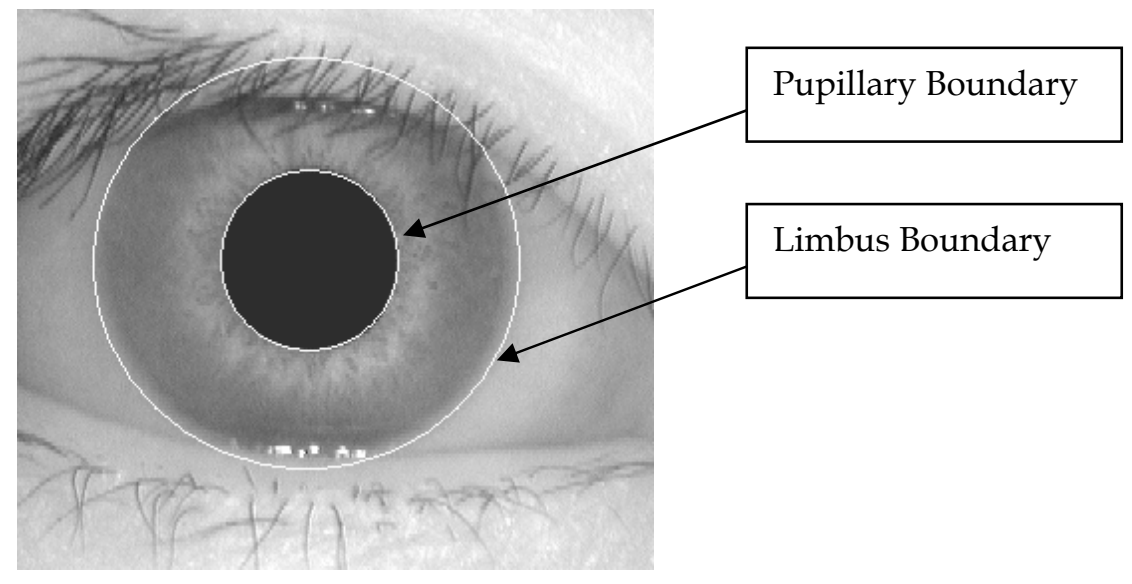

Fig 2.1: Illustration: Iris Segmentation

Fig 2.1 shows an example of iris segmentation. 


\subsection{Motivation}

The main purpose behind our segmentation is to handle the difficulties introduced by the non-ideality of the available images. The approaches currently available in literature do not take these into account completely. There are very few works, which concentrate on the pre-processing module [27-31]. Most of the approaches have compensation for lighting conditions and masking eyelids. They however do not take care of all the possible noises encountered.

Various problems are encountered if the localization methods from the current available approaches are used. Let us take a closer look.

- As shown in Fig 2.2, the pupil may not always be circular. All approaches in current literature [7][10][11][13][14-22] use a circular-based transform for segmentation. This might lead to loss of data and distortions.
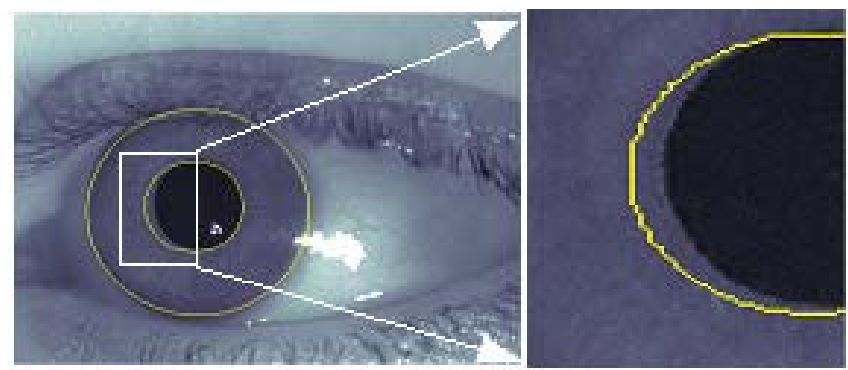

Fig 2.2: Loss of data due to circular-based transform.

- White spots are observed in images due to specular reflections. These also lead to incorrect segmentation. We came across only one technique which takes such spot reflection into consideration, Tisse et. al 's technique [22] proposed in 2002. They however do not take into account any other factors. Illustration in Fig 2.3. 


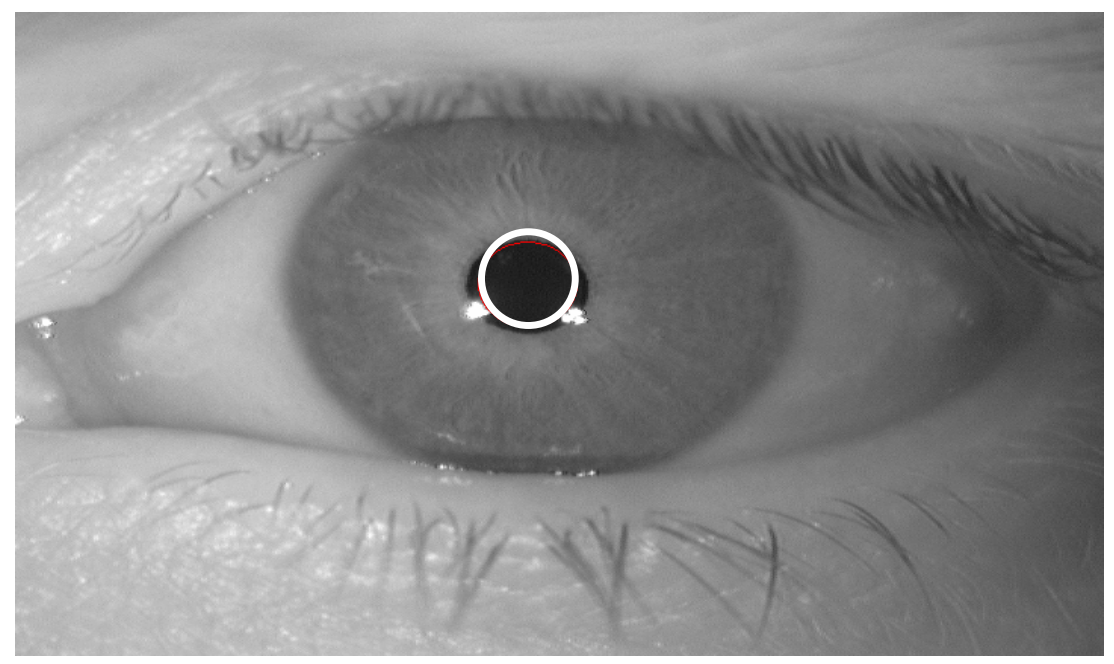

Fig 2.3: Improper segmentation due to specular reflections

- Tuning of thresholds and other parameters has to be done for different databases. We aim to develop an algorithm with minimum tuning of parameters.

We have proposed algorithms for segmenting both these boundaries. The following sections will deal with it in detail. Section 2.2 presents the pupil localization and Section 2.3 deals with the limbus segmentation.

\subsection{Pupil Detection and localization}

The pupil is the opening in the center of the iris. Our task here is to detect the pupil and mark its boundary, which is the inner boundary of the iris pattern. Let us see the proposed algorithm in detail.

\section{Proposed Algorithm}

We aim to take care of the above problems by the proposed "robust" pupil detection algorithm. The algorithm is as follows:

1. Isolating the pupil from the background: 
This is based on a basic assumption that the pupil is darker than the surrounding iris pattern. The contrast between the pupil and the iris texture varies between images, depending upon the characteristics of the user and also due to the change in lighting conditions. We have handled the parameters in such a way that this variation can be accommodated.

The pupil is roughly isolated by binarizing the image. A loose threshold is used here to accommodate varying lighting. Due to this, some portions of eyelashes, which are black, will also be detected here. Fig 2.4 shows the result of this step on an example image. These have to be removed since our required output from this algorithm is just the boundary of the pupil.

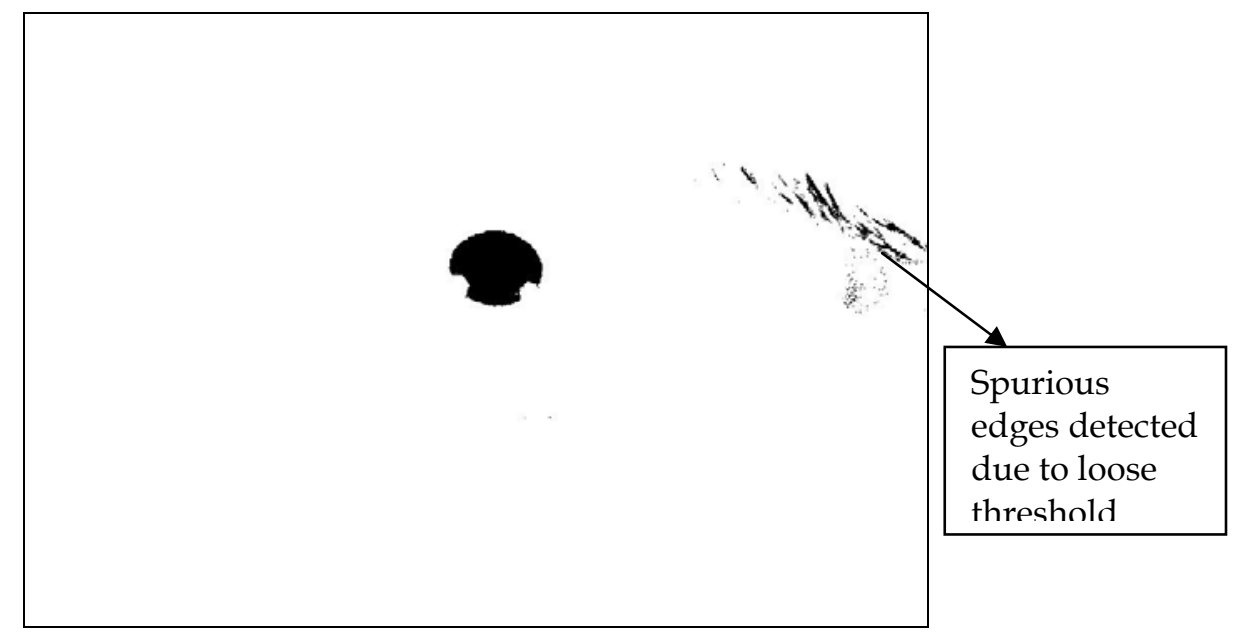

Fig 2.4: Output after binarization

Next, a Canny edge detector [32][33] is used to obtain the boundary of the pupil. The Canny edge detection algorithm is also known as the optimal edge detector. This edge detector first smoothens the image to eliminate noise using a Gaussian filter. It then finds the image gradient to highlight regions with high spatial derivatives. Edges give rise to ridges in the gradient magnitude image. The 
algorithm then tracks along the top of these ridges and sets to zero all pixels that are not actually on the ridge top so as to give a thin line in the output, a process known as non-maximal suppression. The tracking process exhibits hysteresis controlled by two thresholds: $T 1$ and $T 2$ with $T 1>T 2$. Tracking can only begin at a point on a ridge higher than T1. Tracking then continues in both directions out from that point until the height of the ridge falls below $T 2$. This hysteresis helps to ensure that noisy edges are not broken up into multiple edge fragments.

We have used the implementation of the detector available with Matlab. Its usage is as follows: BW = edge (I, 'canny', THRESH, SIGMA) where BW is the output image, I is the input image with the parameters as: THRESH, which is a two-element vector in which the first element is the low threshold, and the second element is the high threshold; and SIGMA is the standard deviation of the Gaussian filter. The output of the edge detector is largely dependent on the choice of sigma. We have chosen a moderately high value of sigma so that only the stronger edges are detected. A few spurious edges will also be obtained here because of the noise due to the eyelids/eyelashes; since they also produce sufficiently strong edges. Using a higher value of sigma cannot solve this problem, since it is important that too many breaks are not introduced in the pupil boundary. Hence, to further isolate the pupil, morphological operations are used to remove the unwanted noise.

The morphological operations [32] consist of two steps; the first is bridging the gaps that might be introduced by the edge detector. This step is necessary to ensure that the edges are continuous. Here also we have used the Matlab implementation of the morphological operation of bridging. Its usage is as follows: BW2 = bwmorph (BW1, OPERATION, N) where BW2 is the output image and BW1 is the input binary image. It applies the operation $\mathrm{N}$ times. $\mathrm{N}$ 
can be Inf, in which case the operation is repeated until the image no longer changes. The operation we have used is 'bridge' for $\mathrm{N}$ as Inf.

The number of connected pixels is used by the next step as a criterion to remove the noise. Only those regions with connected pixels more than a certain value ' $\mathrm{P}$ ', are allowed to remain. This is one of the few parameters in our algorithm that might have to be tuned according to the quality of images, or with change in databases. The usage of the Matlab implementation is as follows:

BW2 = bwareaopen (BW, P) removes from a binary image all connected components (objects) that have fewer than P pixels, producing another binary image BW2. The default connectivity is 8 for two dimensions.

As an example, the output after this step is shown in Fig. 2.5

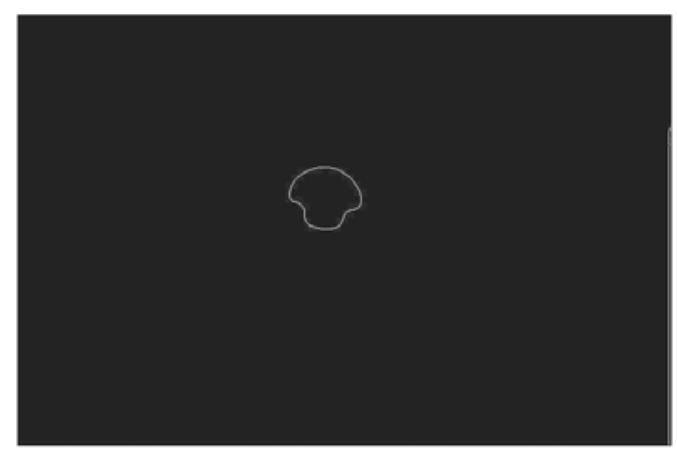

Fig 2.5: Output after morphological processing

2. Masking Specular reflections:

As mentioned before, due to specular reflections, white spots have been observed to appear on images. These lead to incorrect segmentation of the pupil and hence must be masked. A simple thresholding operation is performed with a very high threshold to detect the white spots. To avoid detecting other reflections that may occur on eyelids, a bounding box is created around the localized pupil and white spots are detecting within this area. The area of the iris image 
containing these white spots is masked out and is not considered during further processing. The resulting image after this step is shown in Fig 2.6

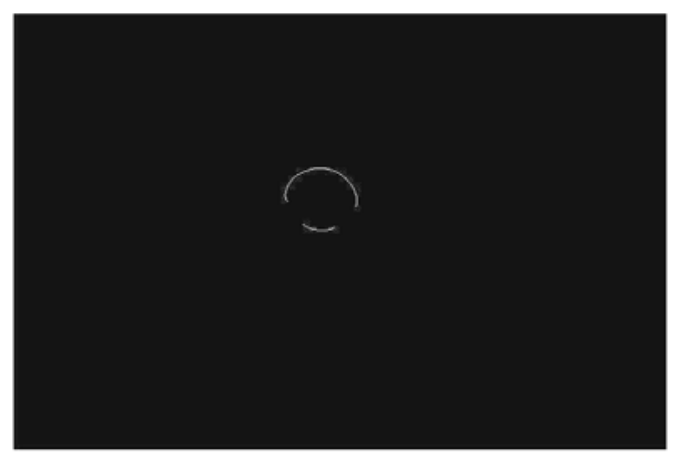

Fig 2.6: Output after step 2 (White boundary- Pupil boundary)

3. Fitting Pupil Boundary with Ellipse:

The pupil boundary locations are then fed into an ellipse-fitting algorithm. The algorithm used here is the Least-squares fit of ellipse to 2D points, presented by Fitzgibbon et al in 1996 [34]. It returns the parameters of the best-fit ellipse to 2D points $(X, Y)$ found in above step. The returned vector contains the center, radii, and orientation of the ellipse. This method combines several advantages:

(i) It is ellipse-specific so that even bad data will always return an ellipse;

(ii) It can be solved naturally by a generalized Eigen system

(iii) It is extremely robust to noise and occlusions and

(iv) It is efficient and easy to implement.

The method uniquely yields elliptical solutions that, under the normalization $4 a c-b^{2}=1$, minimize the sum of squared algebraic distances from the points to the ellipse. This offers a very good trade-off between speed and accuracy for ellipse fitting.

Thus, using Fitzgibbon's algorithm, the pupil boundary is approximated by an ellipse and its parameters such as major and minor axes and centre are obtained. 
These parameters can be used for the further steps in the iris recognition algorithm. Fig 2.7 shows the final result for the example image.

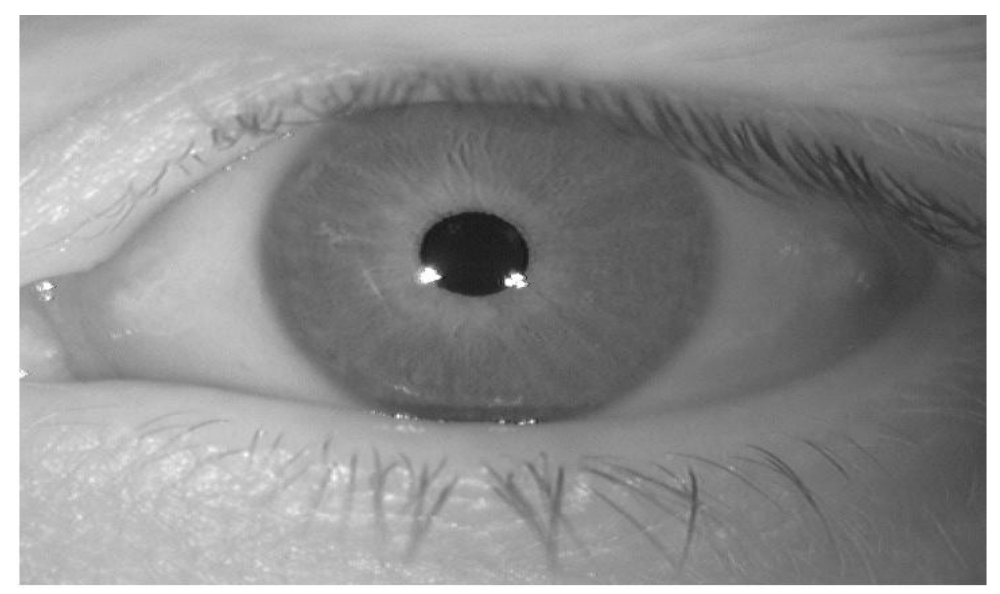

(a) Original Image

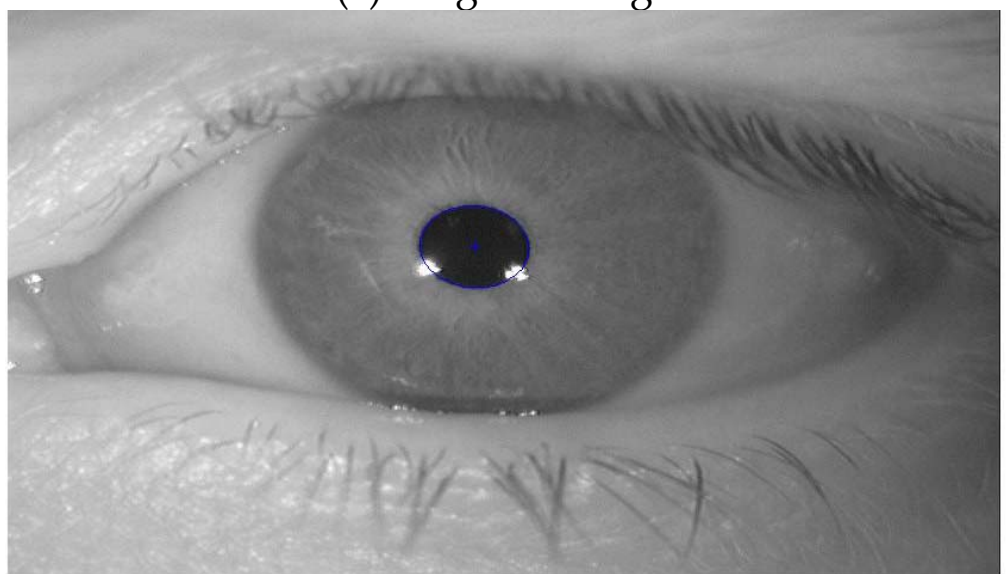

(b) Image after pupil localization

Fig 2.7: Results of Pupil localization

\subsection{Limbus Segmentation}

The next task is the demarcation of the outer or the limbus boundary of the iris. The sclera is commonly known as "the white of the eye." It is the tough, opaque tissue that serves as the eye's protective outer coat. We present our approach to segment the iris from the sclera. Let us see the proposed algorithm in detail.

\section{Proposed Algorithm}


The assumption made here is that the centers of the pupil and iris are the same.

1. Once the pupil is localized, concentric ellipses around it are considered. We plot a function $f(c)$, which is the average intensity, for $c=R / r>1$. $R$ is the outer radius and $r$ is the inner radius. If ' $a$ ' and ' $b$ ' are the major and minor axes of the pupil, the concentric ellipses are selected such that: $a^{`}=a^{*} c^{\prime} ; b^{`}=b^{*} c$, where $a^{`}$ and $b^{`}$ are major and minor axes of the outer ellipses. ' $\mathrm{f}$ ' is calculated for each ellipse and plotted. This plot, for an example image is shown in Fig 2.8.

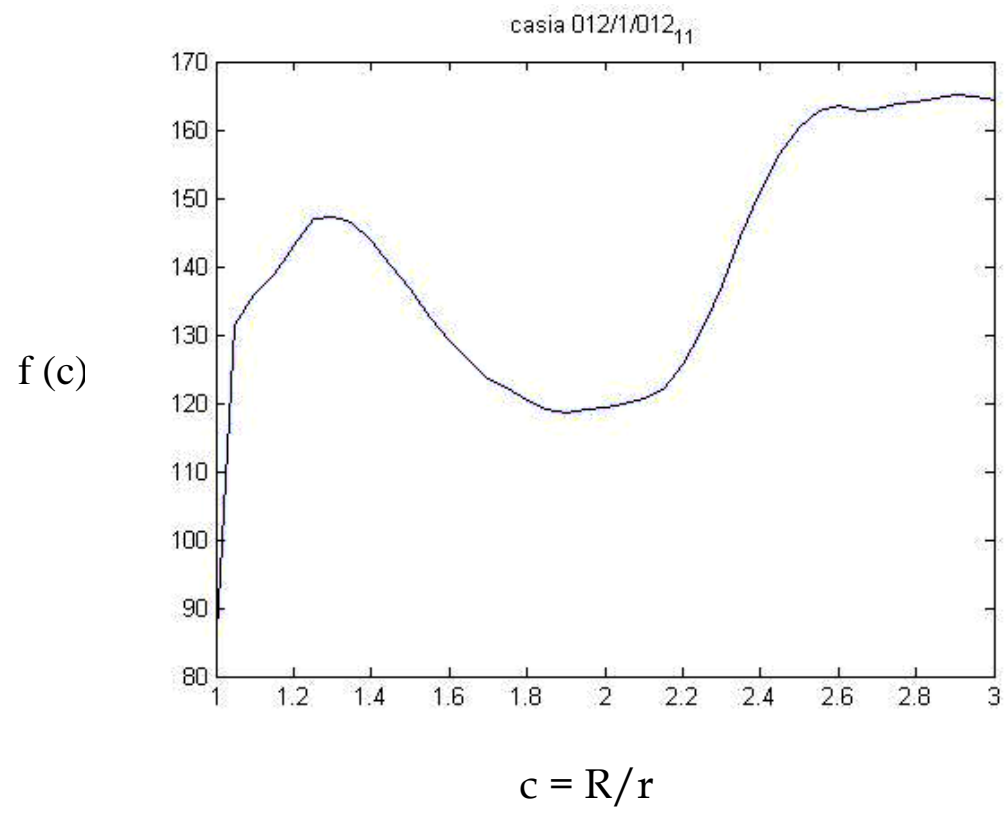

Fig 2.8: Plot for average intensity $\mathrm{f}(\mathrm{c})$

2. We propose that the point on the plot where there is a sudden leap in the value of ' $\mathrm{f}$ ' corresponds to the outer radius. This is because the sclera region is significantly lighter in intensity than the iris pattern, which introduces the leap. This has also been empirically proven. In order to enhance this shoot in value, so as to enable its easy detection, non-linear inverse diffusion [35] is used. 
Non-linear inverse diffusion is recovering the fine-scale signal from its coarse representation. It is an ill-posed problem and gives rise to numerically unstable computational results. [35] presents an analytical solution to the inverse diffusion problem for a specific class of signals. The reason only step edges are considered is that they often characterize the global structure of an image and there exists a precise inverse diffusion solution for this type of edges. A new class of nonlinear diffusion filters is introduced that are conceptually simpler than PDE- based approaches but work perfectly for the step-edge model.

The plot for average intensity after non-linear diffusion is shown in Fig 2.9.

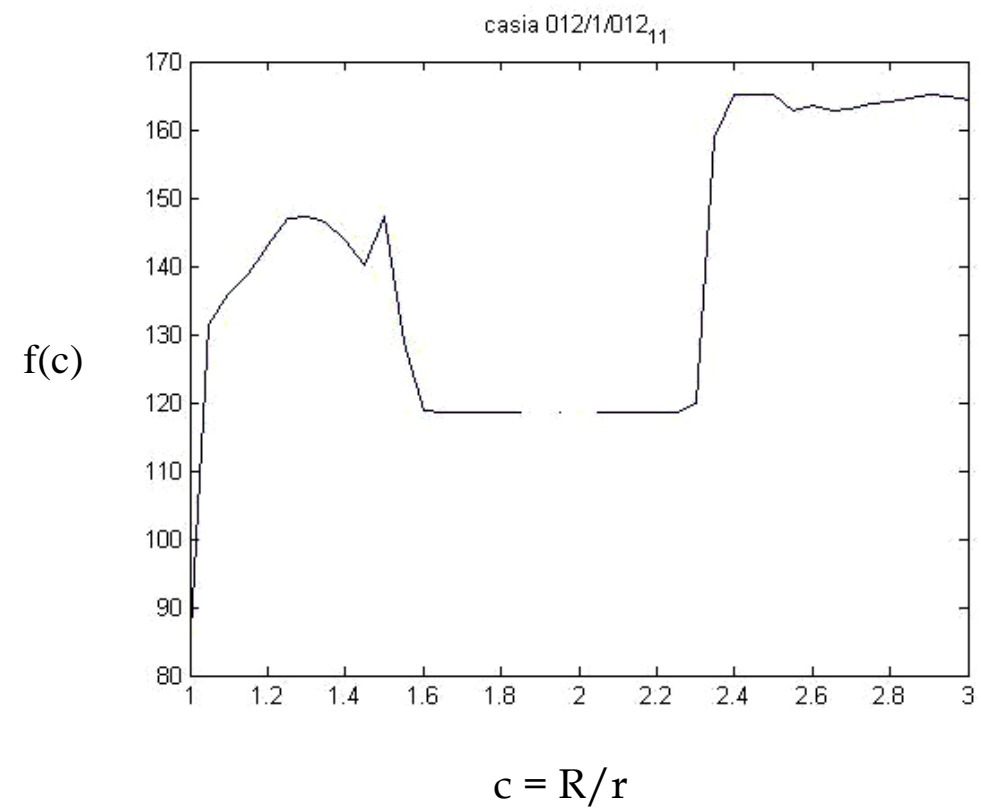

Fig 2.9: Plot for average intensity $\mathrm{f}(\mathrm{c})$ after non-linear diffusion.

An interesting observation made from these plots was that a region of uniform intensity occurs, just before which there is a peak. This peak was observed to be the collarette region of the iris, which is the part of the iris just around the pupil, which is rich in texture. 
3. The algorithm detects this leap, and the outer boundary of the iris is marked according to the value obtained for the outer radius. In the example shown above, the algorithm gave the value of outer radius to be 2.28 times that of the inner radius whereas the ground truth was 2.3. Thus our approach gives a sufficiently accurate value. This can be further seen from the segmentation results shown in the next section.

\subsection{Examples}

The previous sections explained our proposed segmentation algorithm in detail. Let us see some of the results.

As explained before, our algorithm localizes the pupil correctly even if there are white spots due to reflections. Fig 2.10 shows an example of accurate segmentation in spite of specular reflections. Fig 2.11 illustrates correct segmentation even though pupil is not circular.

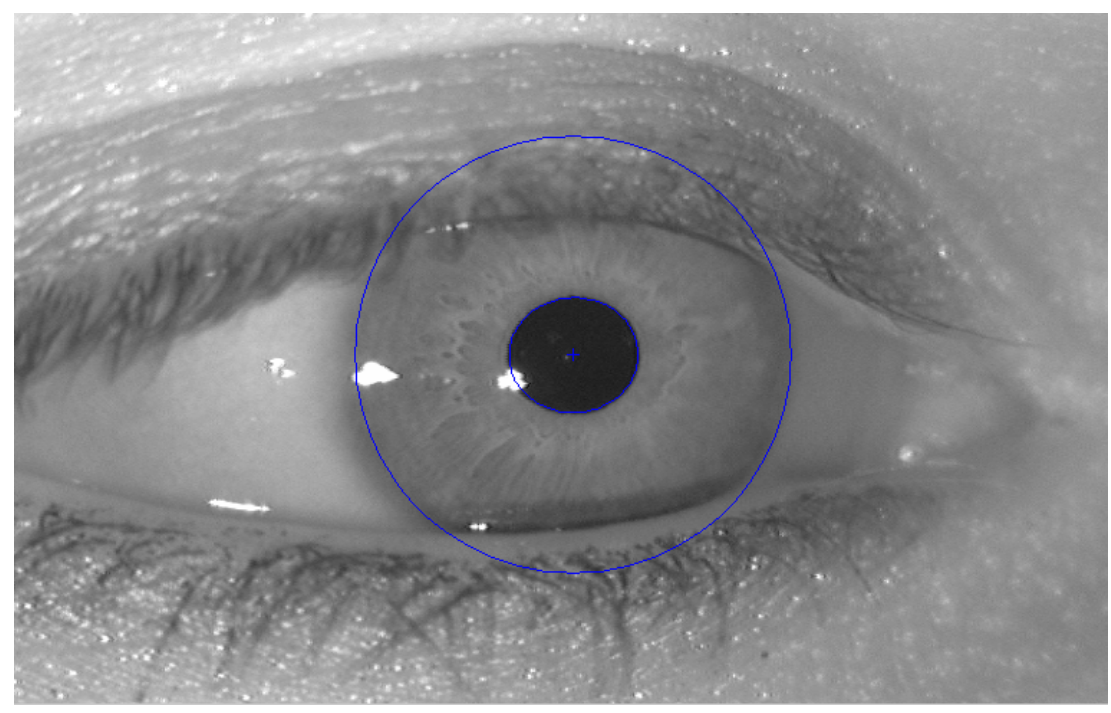

Fig 2.10 Segmentation in presence of specular reflections 


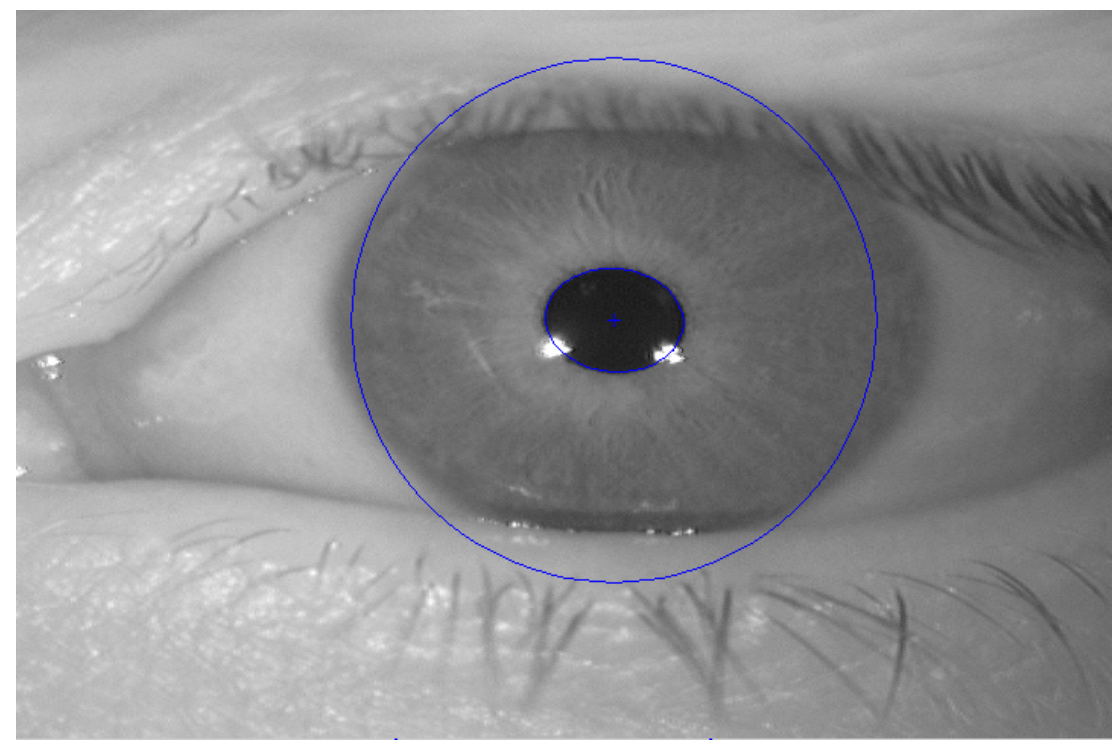

Fig 2.11 Correct segmentation of non-circular pupil

In Fig 2.12 we can see that our algorithm works well for images that are little blurred.

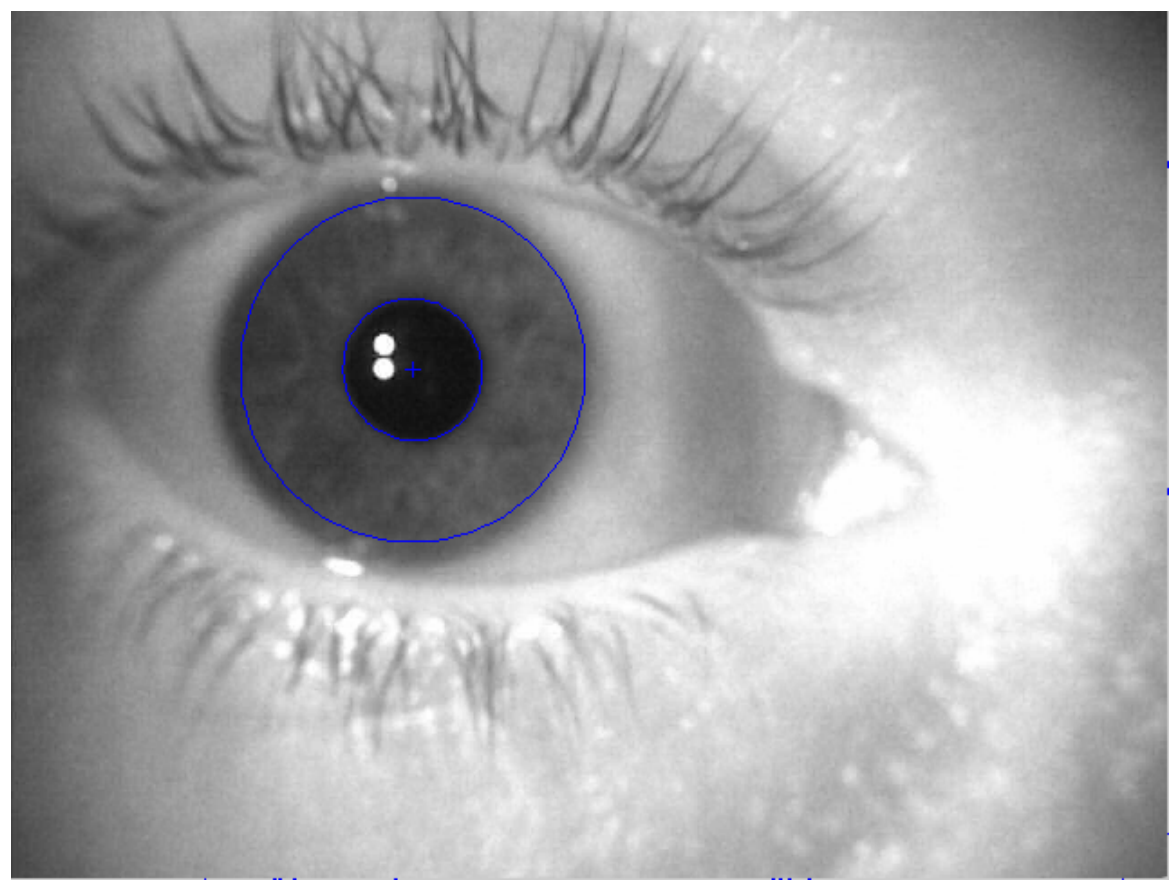

Fig 2.12 Segmentation of blurred image

Our algorithm also accurately segments images occluded by eyelids and eyelashes as shown in Fig 2.13 

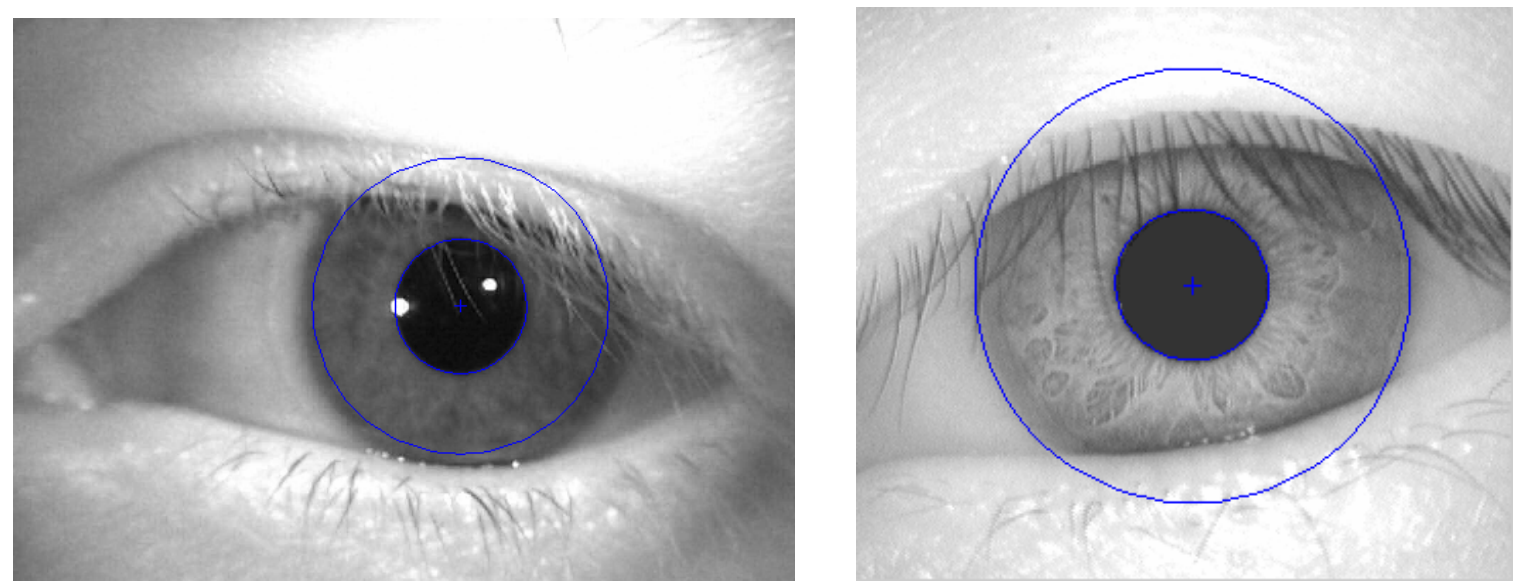

Fig 2.13: Segmentation in presence of occlusions 


\section{Chapter 3}

\section{Dealing with Off-angle Iris Images}

\subsection{Problem Description}

As mentioned before, we are looking to reduce the stringent requirements of iris image capture. A factor that has not been given enough importance to date is the angle of acquisition. In general, most current recognition systems need the user to look "directly" at the camera so as to capture a frontal image. If, however, there is a slight head tilt or rotation, or movement of the eyes in any direction, a slightly different image is presented to the camera.

This is especially important when complete co-operation cannot be expected from the user; like in applications such as hospitals, schools or in surveillance. In such cases, it would prove to be very useful to have the iris recognition software to detect and compensate for this angle. This will play a major role in reducing the intra-class variability introduced by varying angle of acquisition. Fig 3.1 shows examples of the front and off-angle images available. 


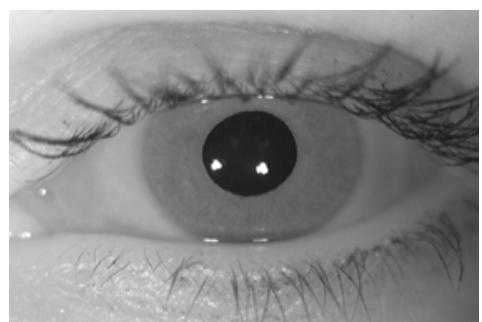

Front Angle

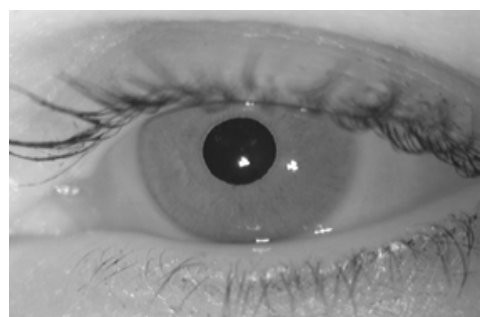

Off- Angle

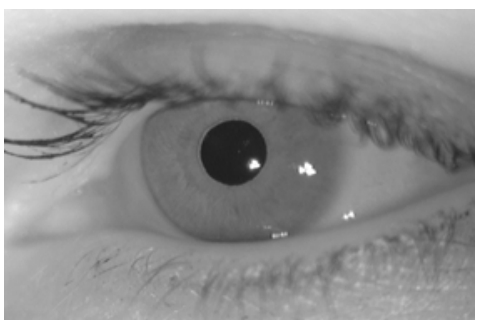

Fig 3.1: Images with varying angle of acquisition

The main difficulty in compensating images is that the angle between the axis of the camera and the eye is unknown. Our first task is hence to estimate this angle from the captured image. The distortion in the off-angle image could be due to a head tilt in either horizontal or vertical direction. Thus, in order to avoid the illposed nature of this problem, we make certain assumptions:

(1) Gaze direction is constant.

(2) Camera is placed at a fixed height above the ground level; thus ensuring that the distortion produced due to varying angle is only along the horizontal direction.

Fig 3.2 illustrates the scenario of image capture.

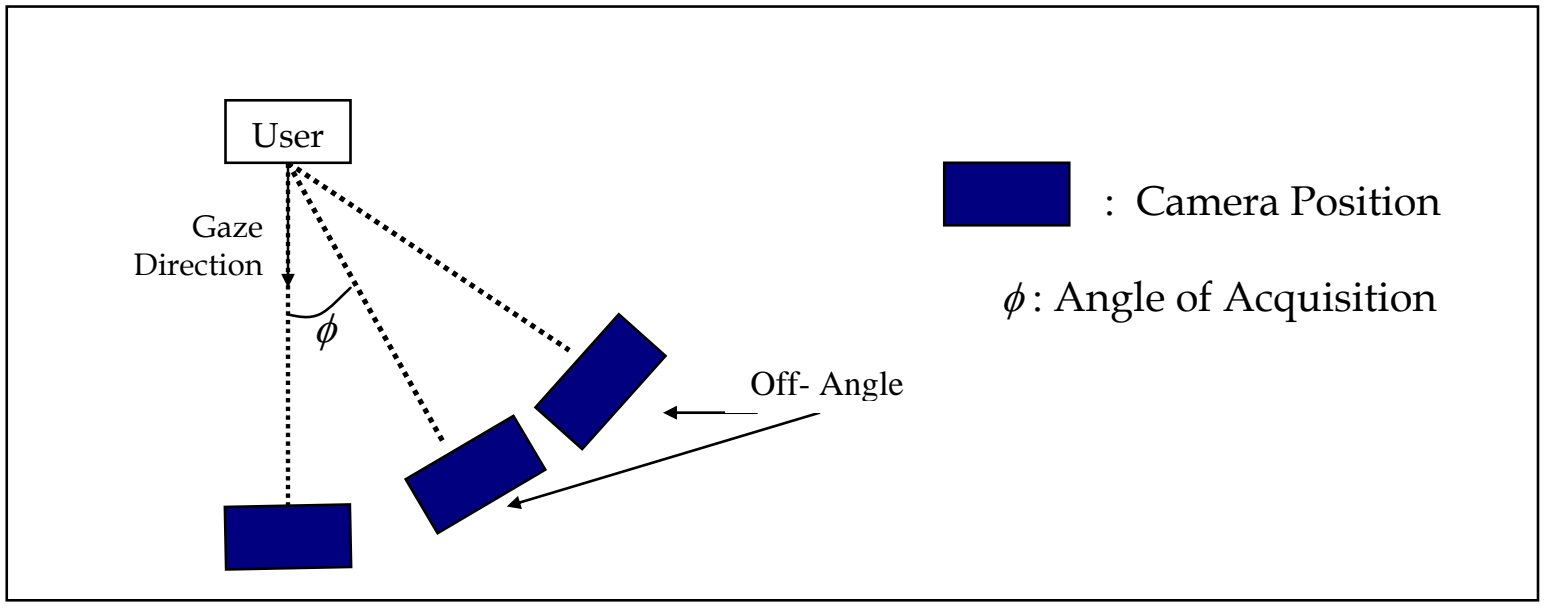

Fig 3.2: Scenario of Image Capture 
In-plane rotations have been taken care of in most of the recognition algorithms, hence we did not feel the need to address that issue.

The motivation behind our proposed algorithm is to compensate all captured images so as to get them as close to their front angle images as possible. A point to be noted here is that we have made an assumption for segmentation that the pupil and iris are concentric. In case of off-angle images with large angles, this may result in a few errors in segmentation. The next section will, in detail, explain our approach for estimating and compensating the angle. Lastly in this chapter we present a few example images before and after compensation.

\subsection{Proposed Solution}

\subsubsection{Angle Estimation}

The scenario for image capture has been explained before. The angle in the 'offangle' images is unknown; hence the first task here is to estimate this angle. Our proposed algorithm is designed to provide a rough estimate of the angle. What we suggest to do is in the recognition stage, have the matching algorithm use this as a coarse initial estimate. Then, the final value of the angle should be decided by finding the matching distances around this coarse estimate, and assigning that angle which gives the minimum distance. Fig 3.3 illustrates the idea.

The search region could be around 5-10 on either side of the estimated angle. It was mentioned before that the segmentation of the pupil using ellipse fitting serves a dual purpose. Not only does it provide a better segmentation to noncircular pupils, it is also useful in estimating the angle. The main assumption we are making is that the pupil is a rough circle in the front view. 


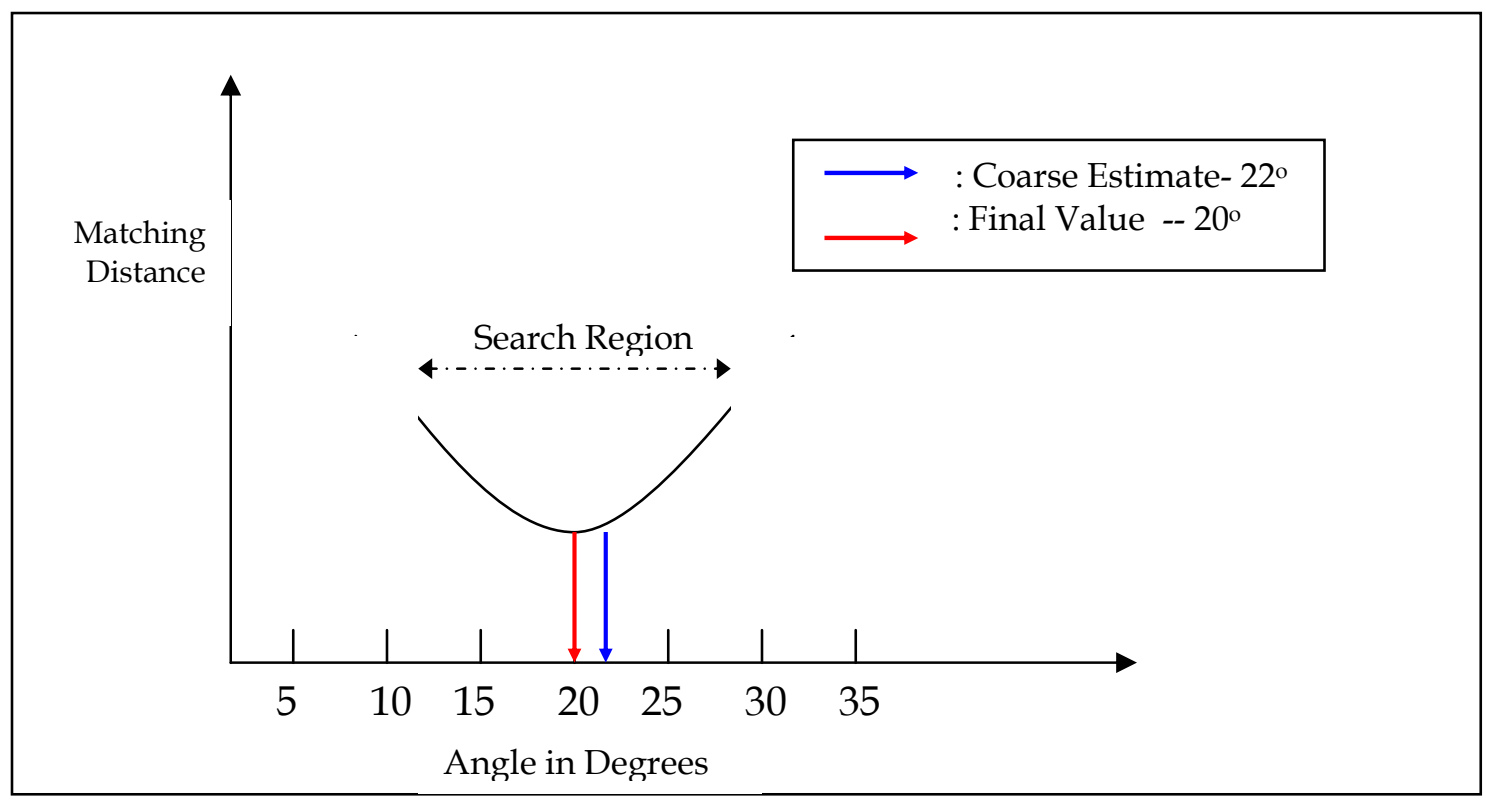

Fig 3.3: Coarse to fine approach for angle estimation

Let us see the proposed algorithm in detail:

\section{STEP 1:}

As explained in the previous chapter, the pupil boundary is fitted to an ellipse and the parameters of the ellipse i.e. the major and minor axes radii and the centre are obtained.

\section{STEP 2:}

Having obtained the ellipse parameters, the angle is estimated as follows:

Fig 3.4 shows the image capture setup. $f 1(x, y)$ denotes the frontal image and $f 2(x, y)$ denotes the off-angle image and $\phi$ is the angle of capture. Referring to Fig. 3.4 , we can say that, assuming fixed gaze direction, capturing an iris image offangle will only affect the dimensions of the iris in horizontal direction. If we assume that the pupil will be circle in the front angle and an ellipse in the off- 
angle, the ratio of the dimensions of the pupil in the horizontal direction can be seen to be the cosine of the angle of acquisition. Refer Fig 3.5.

Thus,

$$
\left[\begin{array}{l}
x \\
y
\end{array}\right]=\left[\begin{array}{cc}
\cos \varphi & 0 \\
0 & 1
\end{array}\right]\left[\begin{array}{l}
x^{\prime} \\
y^{\prime}
\end{array}\right]
$$

Hence, the estimated angle will be:

$$
\hat{\varphi}=\cos ^{-1}(d 1 / d)
$$

where $\mathrm{d} 1=$ minor axis in frontal image and

$\mathrm{d} 2=$ minor axis in off-angle image

However, since we have assumed dimensions of pupil along vertical axis remain constant, this equation is equivalent to:

$$
\hat{\varphi}=\cos ^{-1} \text { (MinorAxis / MajorAxis) }
$$

where the dimensions of pupil in off-angle image only are considered.

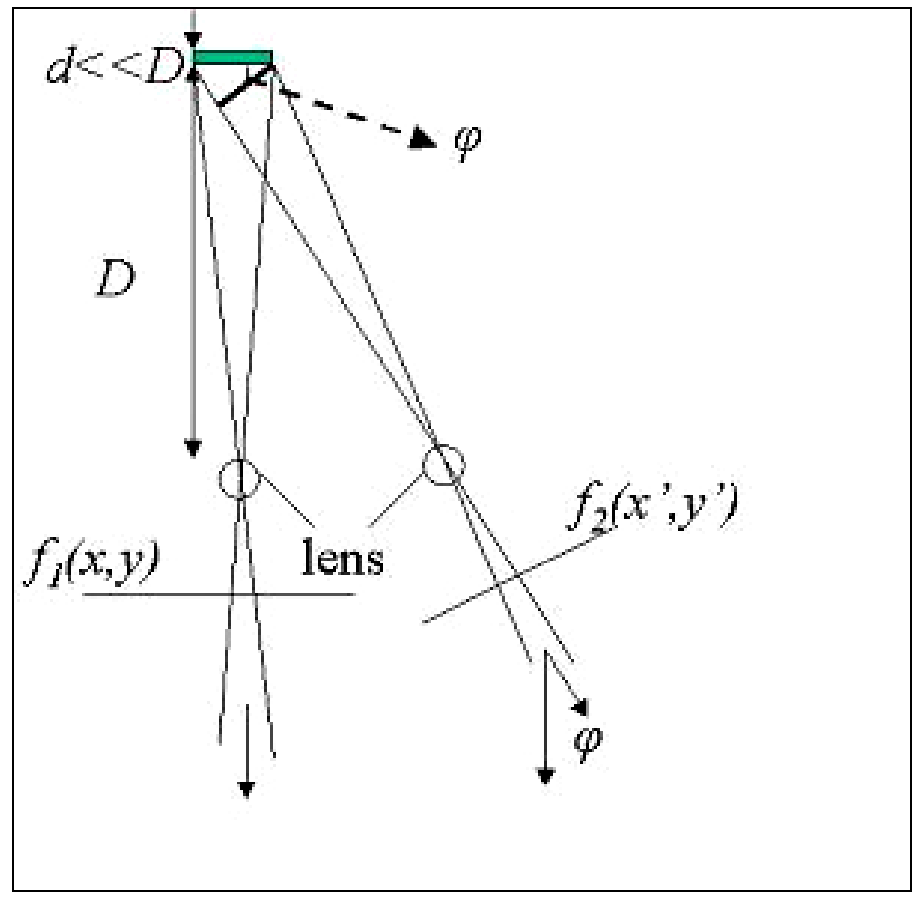

Fig 3.4: Setup for image capture ( $\phi$ : Angle of acquisition) 


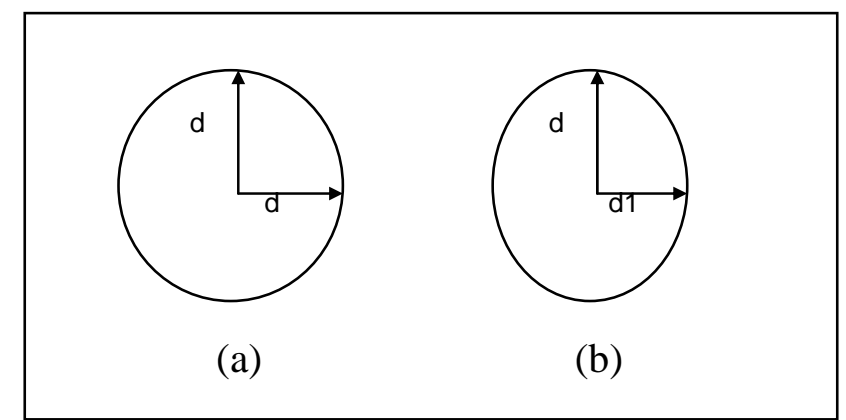

Fig 3.5: Approximation of pupil as circle in (a) Front Angle and as ellipse in (b) Off-Angle

This will be used as a coarse estimate by the recognition stage. Since we take the ratio of major and minor axis for only one view, the angle estimation is independent of the front angle image. This is possible because of the assumption that the scene depth is constant. If there is a change in scene depth this can be easily handled by attributing it to a scaling factor between the two views.

\subsubsection{Angle Compensation}

Our aim is to convert the off-angle image back to a frontal view image. Thus, the next stage after estimating the angle will be to compensate the captured image for that particular angle. As said before, we have approximated the pupil as a circle in the front angle and as an ellipse in the off-angle. The simplest way to convert an ellipse to a circle is by projective transformations.

A projective transformation is a transformation used in projective geometry: it is the composition of a pair of perspective projections. It describes what happens to the perceived positions of observed objects when the point of view of the observer changes. Projective transformations do not preserve sizes or angles but do preserve incidence and cross-ratio. Fig 3.6 illustrates projective transformation. 
We have used the Matlab implementation of projective transformation. The function used is: $\mathrm{T}=$ MAKETFORM ('projective', $\mathrm{U}, \mathrm{X}$ ) builds a TFORM structure for a two-dimensional projective transformation that maps each row of $U$ to the corresponding row of $\mathrm{X}$. $\mathrm{U}$ and $\mathrm{X}$ are each 4-by-2 and define the corners of input and output quadrilaterals. No three corners may be collinear.

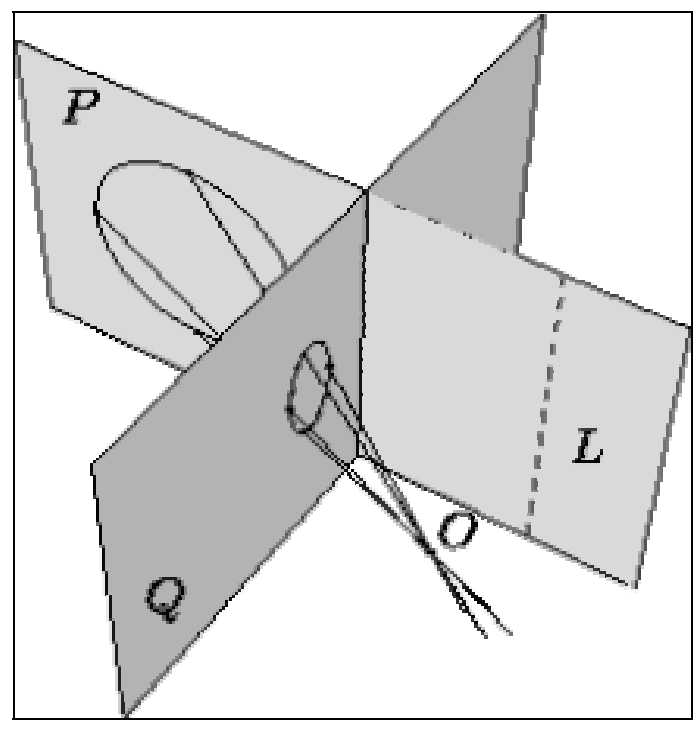

Fig 3.6: A perspective transformation with center $O$, mapping the plane $P$ to the plane $Q$.

$\mathrm{B}=$ IMTRANSFORM (A, TFORM, INTERP) transforms the image A according to the 2-D spatial transformation defined by TFORM, which is a tform structure as returned by MAKETFORM;INTERP specifies the form of interpolation to use. INTERP can be one of the strings 'nearest', 'bilinear', or 'bicubic'. We have used bicubic interpolation.

\subsubsection{Examples}

This section gives a few examples of the results produced after applying our proposed angle estimation-compensation algorithm. Since off-angle images were available only for the Eye Institute Database, the results shown are only for this database. 
As said before, there were two front angle and two off-angle images available for each class. The next chapter shows the overall results for the database. Fig 3.7 shows the original image and the images before and after compensation.

A very important point should be noted here: To take into account the noncircularity of the pupil, we have subjected the front angle as well as the off-angle images to our algorithm. Essentially what we are doing in compensation is making a non-circular pupil circular. In case the front angle does not contain a circular pupil, our algorithm could result in erroneous results, since it will again increase the intra-class distance, which is not desired. Subjecting all the images to our algorithm will thus bring them to a common level so that they can be compared against each other without adversely affecting the intra-class distance. Fig 3.8 shows similar results for a different user.

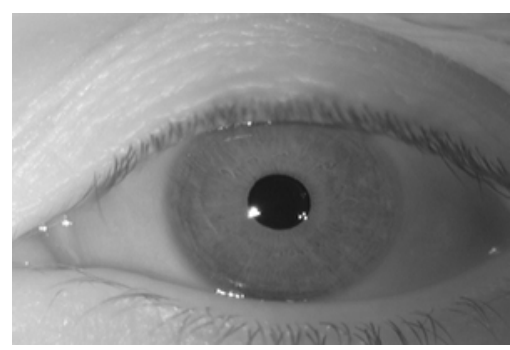

Front angle

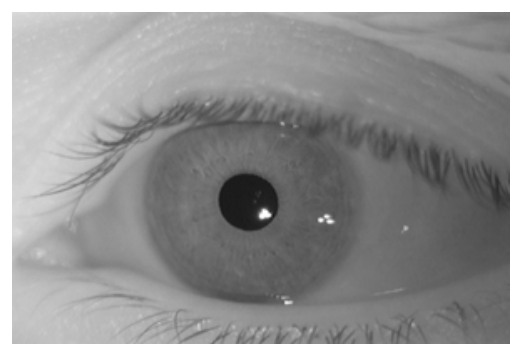

Off Angle (1)

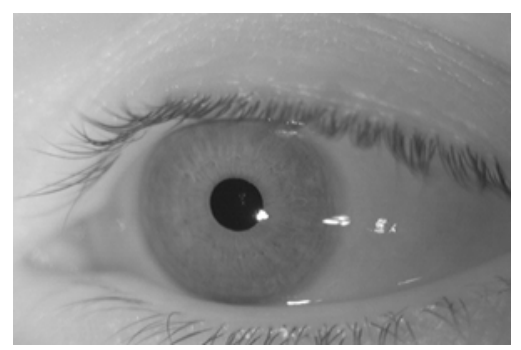

Off Angle (2)

(a) Original Images
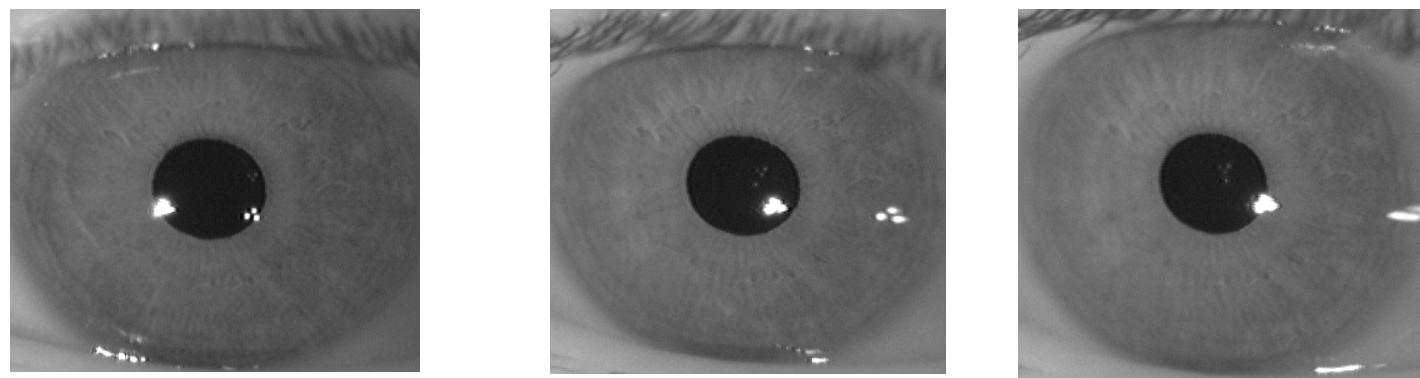

(b) Images after Angle Estimation-Compensation

Fig 3.7: Compensation Results (User 1) 


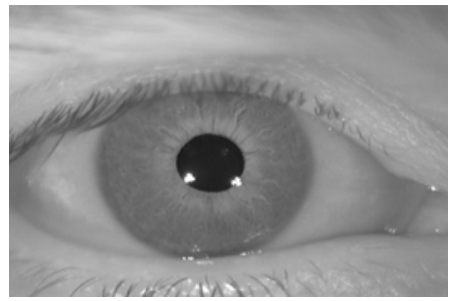

Front Angle

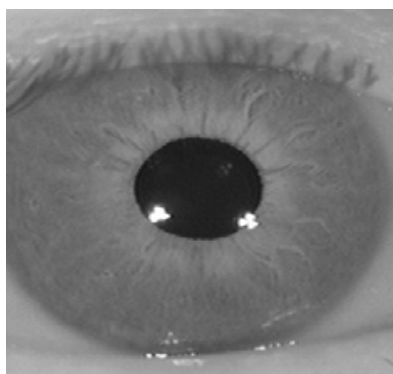

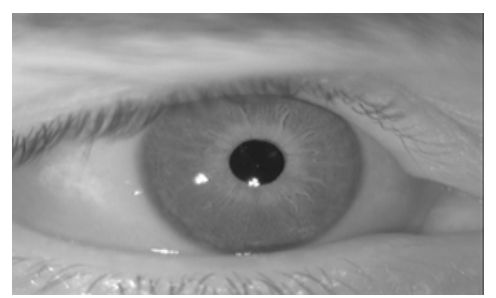

Off Angle (1)

(a) Original Images

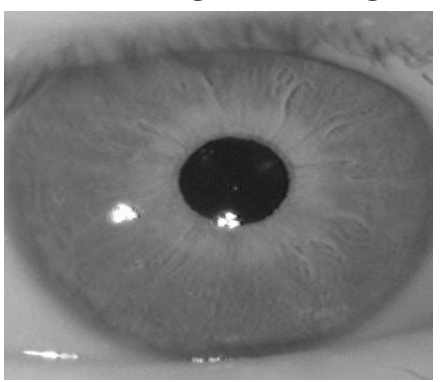

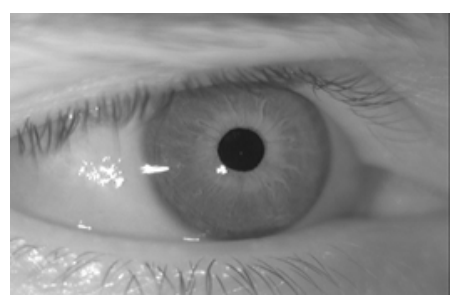

Off Angle (2)

(b) Images after Angle Estimation-Compensation

Fig 3.8: Compensation Results (User 3)

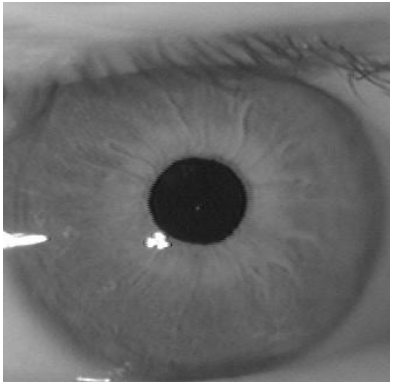




\section{Chapter 4}

\section{Results}

Since our objective in this work is to effectively segment the iris pattern from the entire image, the results can be better appreciated by visual inspection. Here we have only evaluated the performance of our pre-processing algorithm. Another way of performance evaluation could be to use our segmentation algorithm before any popular matching technique and inspect the ROC Curves. But the matching performance will also affect the overall results; hence it will be difficult if not impossible to evaluate the segmentation.

Though we can visually inspect each segmented image, we thought it imperative to devise a way to evaluate the overall performance, across different images and databases. To enable this, we assigned scores to the resulting images depending on how successful the segmentation is. Scores were assigned as shown in Table 2.

Depending on the number of images available in each database, we selected a few representative images and ran our proposed algorithm on these images. While selecting the images to test our algorithm, such images were chosen which conformed to the assumptions made. The results thus show segmented images for only a small subset of the entire database. As mentioned in Section 1.2, we had four different databases available. These were varying in image acquisition 
conditions; hence it proved to be very challenging to obtain good results on all databases with minimum tuning of parameters.

Table 3 shows the success rate calculated for all the databases.

\begin{tabular}{|c|c|c|}
\hline Score & $\begin{array}{c}\text { Pupil boundary } \\
\text { segmentation }\end{array}$ & $\begin{array}{c}\text { Limbus Boundary } \\
\text { segmentation }\end{array}$ \\
\hline 10 & Excellent & Excellent \\
\hline 9 & Good & Good \\
\hline 8 & Excellent/Good & Fair \\
\hline 8 & Fair & Excellent/Good \\
\hline 5 & Incorrect & $X$ \\
\hline 5 & $X$ & Incorrect \\
\hline 0 & Incorrect & Incorrect \\
\hline
\end{tabular}

Table 2: Scoring Mechanism (X - Don't Care)

\begin{tabular}{|l|c|c|}
\hline & Total \# of Images Tested & Success Rate \\
\hline CASIA Database & 60 (10 users * 6 per user) & $95.2 \%$ \\
\hline Eye Inst. Database & 40 (10 users * 4 per user) & $96.0 \%$ \\
\hline WVU Database & 20 & $93.5 \%$ \\
\hline UB Iris Database & 50 (10 users * 5 per user) & $89.8 \%$ \\
\hline
\end{tabular}

Table 3: Overall Results of proposed algorithm

The success rate is calculated as follows:

Score $=$ Total score obtained / $(\#$ images tested $* 10)$

Success Rate $=($ Score $* 100) \%$

A point to be noted here is that since we have assigned a score of 10 only to perfect segmentation results and 9,8,5 to slightly erroneous ones. Hence a success rate of $95 \%$ does not necessarily mean that our algorithm cannot segment $5 \%$ images. 
The highlight of our algorithm is it does not require extensive tuning of parameters. There are three main parameters, which need to be assigned initial values according to the nature of the database. This can be done by manually inspecting the images obtained from the database under consideration.

a. Threshold: This is required for the rough binarization of the image in the first stage as explained in Chapter 2.

b. Pixel Count: Depending on the lighting conditions for the database, the size of the pupil will change. The pixel count is the lower limit on the size of the pupil.

c. Ratio: As the size of the pupil changes with light, the ratio of the radius of the pupil to the limbus radius also changes. The ratio parameter is the upper limit for the same, which helps determine the search area for the limbus boundary.

Tuning these parameters is very simple, unlike in other contemporary algorithms.

The further sub sections give the details about the performance of the algorithm for each of the four databases.

\subsection{CASIA Database}

The CASIA Database was collected by National Laboratory of Pattern Recognition (NLPR), Institute of Automation (IA) and the Chinese Academy of Sciences (CAS) to promote research. This database (Version 1.0) includes 756 iris images from 108 eyes (hence 108 classes). For each eye, 7 images are captured in two sessions, where three samples are collected in the first session and four in the second session. The images are stored as: $X X X \backslash S \backslash X X X \_S \_Y . b m p$, where $X X X$ is the unique identifier of eye, ranging from 000 to $108, S$ is the index of session and $Y$ is the index of image in the same session. ' 1 'denotes the first session and ' 2 'the second session. $Y$ ranges from 1 to 3 in the first session, 1 to 4 in 
the second session. Therefore XXX_S_Y.bmp means the Yth iris image taken in session $S$ from eye $X X X$. We selected 10 representative users from the database and ran our algorithm for 6 images of each user.

This database consists of all frontal images, and there are no specular reflections in any of the images. Hence this database gives very good results. The factors leading to problems in segmentation are that many images have large percentage of occlusions due to eyelashes/ eyelids. Also, the sclera region is observed to be grayish, which introduces difficulty in properly defining the limbus boundary.

Table 4 gives the number of images for each assigned score. Fig 4.1 shows examples of results for each score obtained.

\begin{tabular}{|c|c|}
\hline Score & \# Of images \\
\hline 10 & 49 \\
\hline 9 & 7 \\
\hline 8 & 11 \\
\hline 5 & 0 \\
\hline 0 & 0 \\
\hline
\end{tabular}

Table 4: Scores for CASIA Database

Total images tested $=60$

Total score obtained $=10 * 49+9 * 7+8 * 11+5 * 0=571$

Score $=571 /(60 * 10)=0.952$

Success Rate: 95.2 \% 


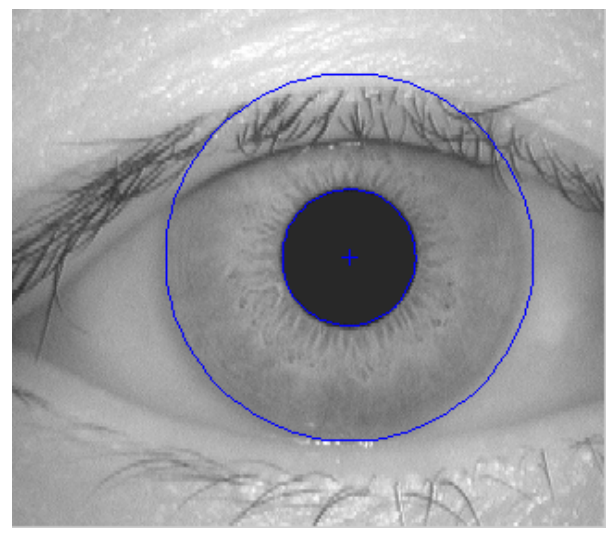

(a) Score: 10

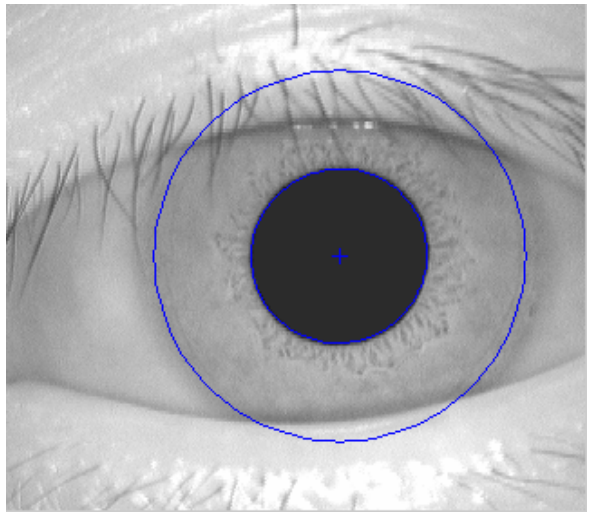

(b) Score: 9

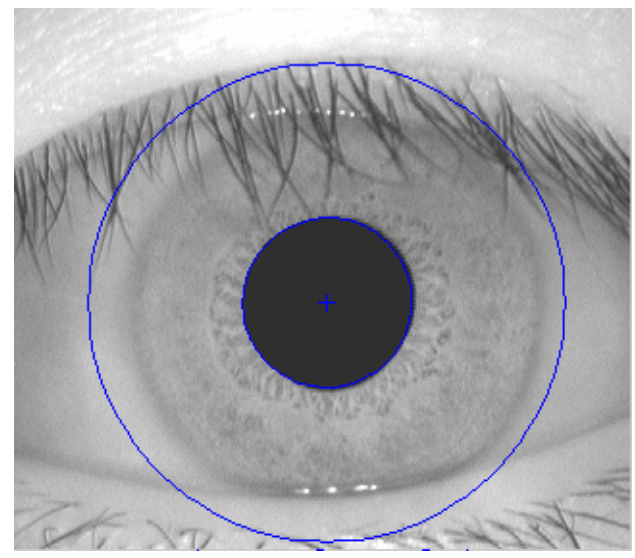

(c) Score: 8

Fig: 4.1: Segmentation Results for CASIA Database

\subsection{Eye Institute Database}

Dr A. Realini and Dr J. Realini at the WVU Eye Center were involved in collection of this database. It consists of about 100 users and 8 images per user. There are 4 images per eye i.e. 4 images per class. For each eye, two images are front angle and two are off-angle.

We selected 10 users and 4 images per user and tested our algorithm. Since this database consisted of off-angle images, the angle of the off-angle images was estimated and the images were compensated by that angle. The factors that introduced non-ideality in the iris images in this database were specular reflections, occlusions and varying angle of acquisition. 
Table 5 shows the results. Fig 4.2 shows example images for each score obtained.

\begin{tabular}{|c|c|}
\hline Score & \# of images \\
\hline 10 & 30 \\
\hline 9 & 7 \\
\hline 8 & 2 \\
\hline 5 & 1 \\
\hline 0 & 0 \\
\hline
\end{tabular}

Table 5: Scores for Eye Institute Database

Total images tested $=40$

Total score obtained $=10 * 30+9 * 7+8 * 2+5 * 1=384$

Score $=384 /(40 * 10)=0.960$

Success Rate: $96 \%$

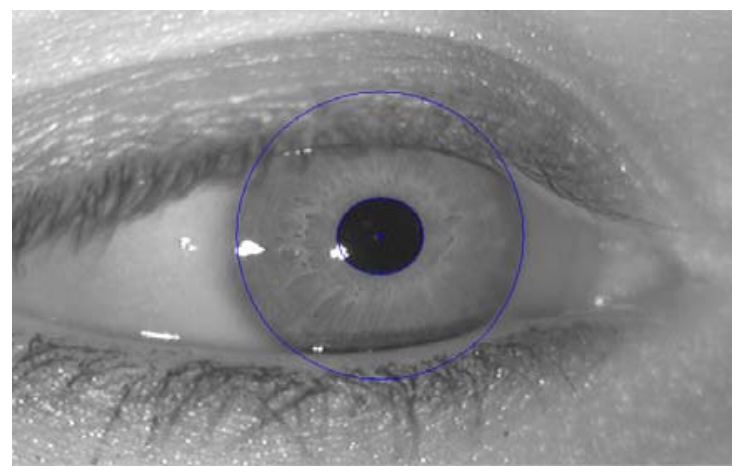

(a) Score : 10

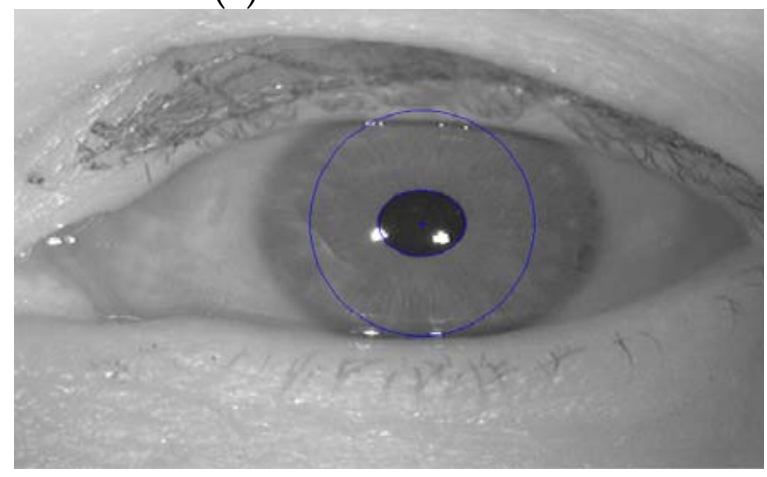

(c) Score: 8

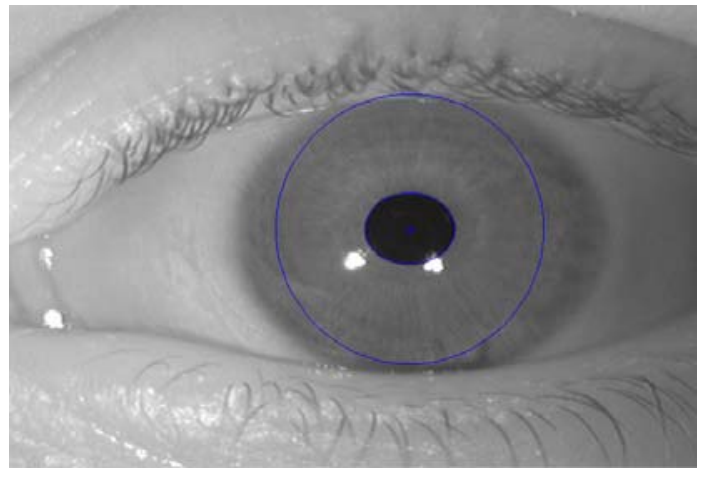

(b) Score: 9

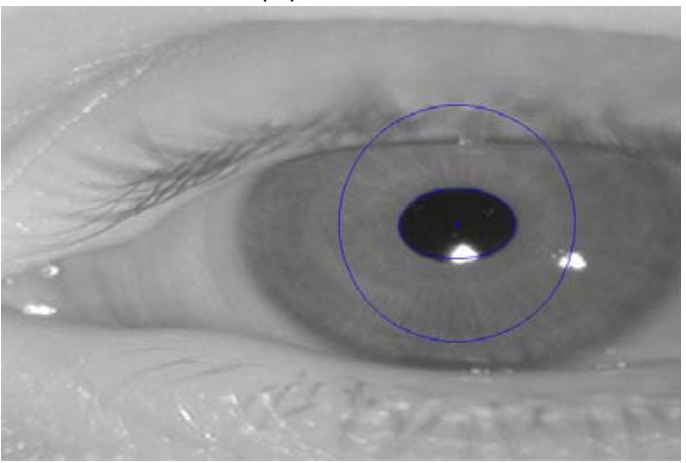

(d) Score: 5

Fig: 4.2: Segmentation Results for Eye Institute Database 


\subsection{WVU Database}

This database was captured at West Virginia University to facilitate availability of non-ideal images for the "Non-Ideal Iris Recognition" Project. This was thus the most challenging database since it contains images that are of varying lighting conditions, with specular reflections and some are blurred. In spite of this, it should be noted that there are no images with scores of 5 or 0 , thus there were no actual failures in segmentation. Table 6 shows the number of images for each score. Fig 4.3 shows example images for each score obtained.

\begin{tabular}{|c|c|}
\hline Score & \# of images \\
\hline 10 & 12 \\
\hline 9 & 3 \\
\hline 8 & 5 \\
\hline 5 & 0 \\
\hline 0 & 0 \\
\hline
\end{tabular}

Table 6: Scores for WVU Database (1)

Total images tested $=20$

Total score obtained $=10 * 12+9 * 3+8 * 5+5 * 0=187$

Score $=187 /(20 * 10)=0.935$

Success Rate: 93.5 \% 


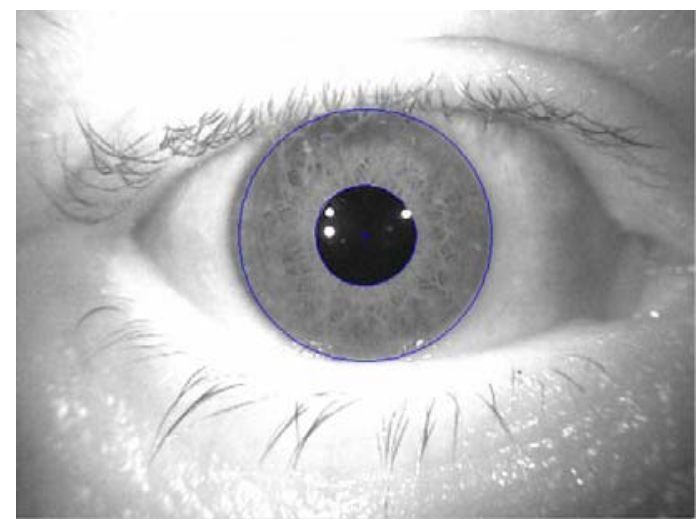

(a) Score: 10

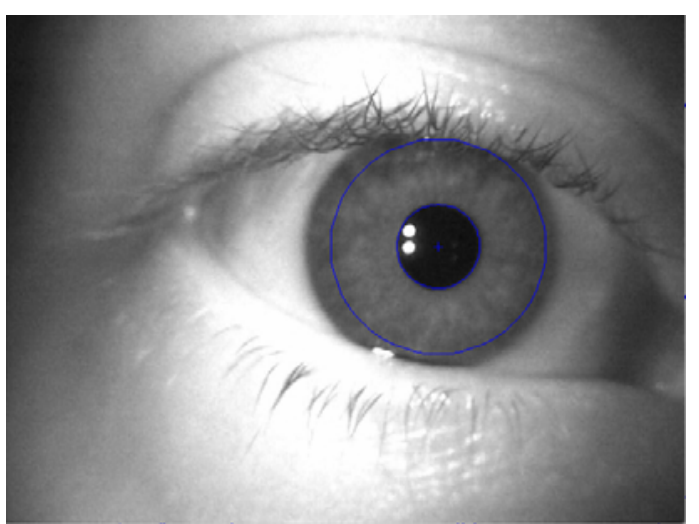

(b) Score: 9

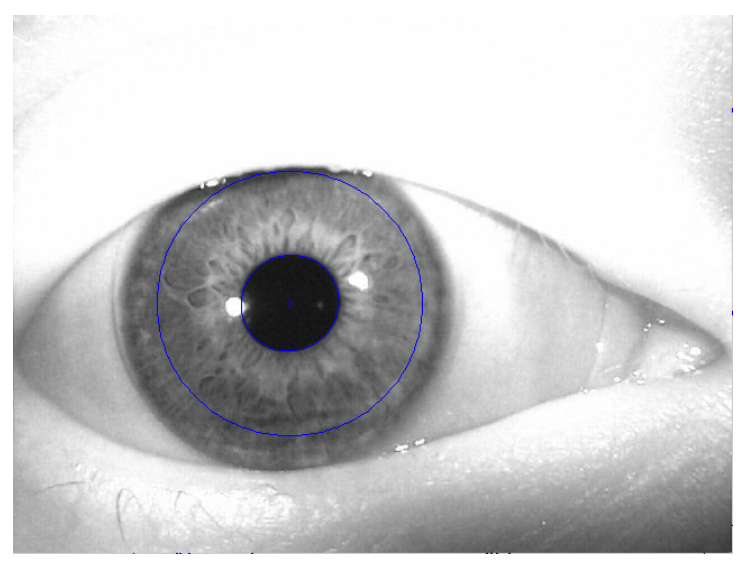

(c) Score: 8

Fig 4.3: Segmentation Results for WVU Non-Ideal Database

\subsection{UB Iris Database}

UBIRIS database [36] is composed of 1877 visible light images collected from 241 persons during September 2004 in two distinct sessions. Its main characteristics result from the fact that, in opposition to the existing public and free databases (CASIA and UPOL), it incorporates images with several noise factors, thus permitting the evaluation of robustness iris recognition methodologies.

It is available at http://iris.di.ubi.pt.

The difficulty in segmenting images from this database was that there was very less contrast between the pupil and the iris texture. This led to inaccurate binarization results, which in turn affected all the further steps in the algorithm. 
Also, the specular reflections were very large as compared to the size of the pupil in almost all cases. In many images, more than $50 \%$ of the pupil was white due to specular reflections. This also gave a few erroneous results. Contrary to most databases, here the limbus boundary was more defined and hence there were fewer errors in segmenting the iris pattern from the sclera. Table 7 shows the number of images for each score. Fig 4.4 shows example images for each score obtained.

\begin{tabular}{|c|c|}
\hline Score & \# of images \\
\hline 10 & 34 \\
\hline 9 & 3 \\
\hline 8 & 6 \\
\hline 5 & 5 \\
\hline 0 & 1 \\
\hline
\end{tabular}

Table 7: Scores for UB Iris Database

Total images tested $=50$

Total score obtained $=10 * 34+9 * 3+8 * 6+5 * 5=440$

Score $=440 /\left(490^{*}\right)=0.898$

Success Rate: $89.8 \%$

* One image consisted of closed eye, which was removed. 


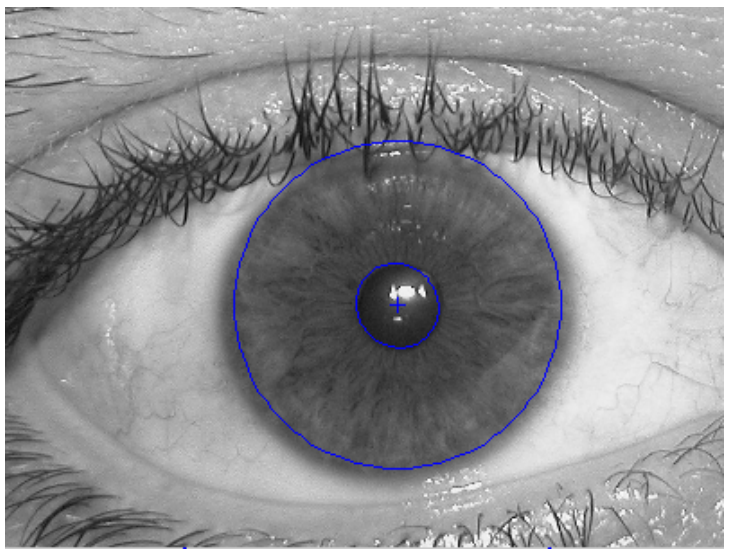

(a) Score: 10

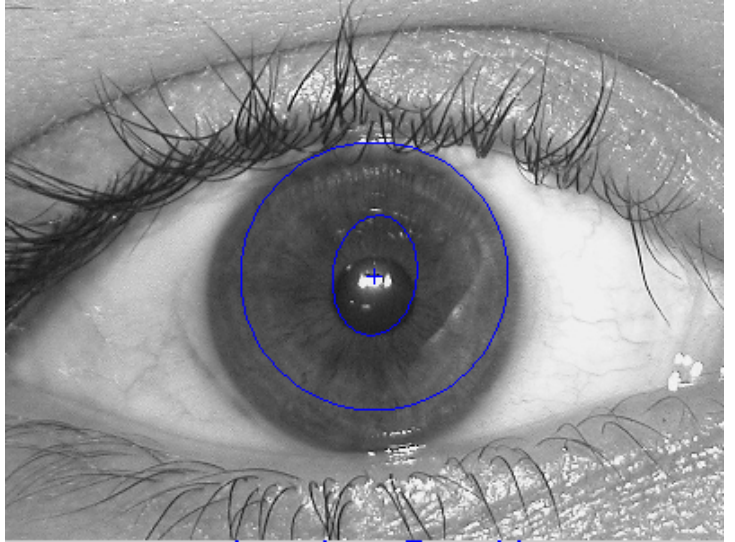

(c) Score: 8

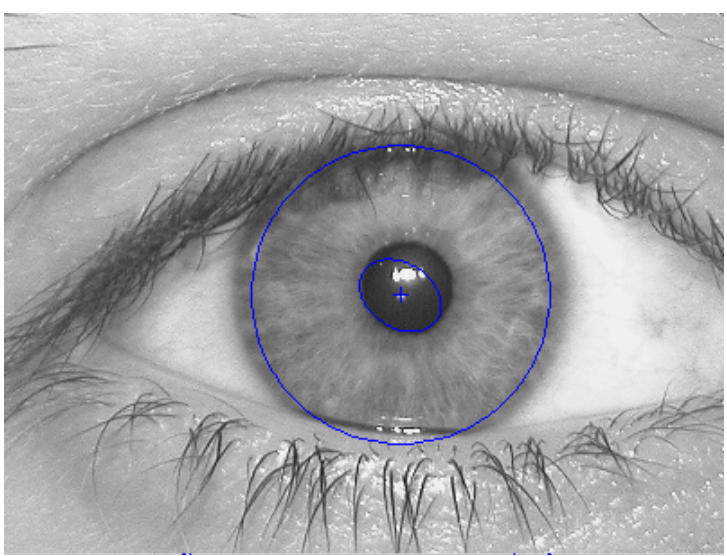

(b) Score: 9

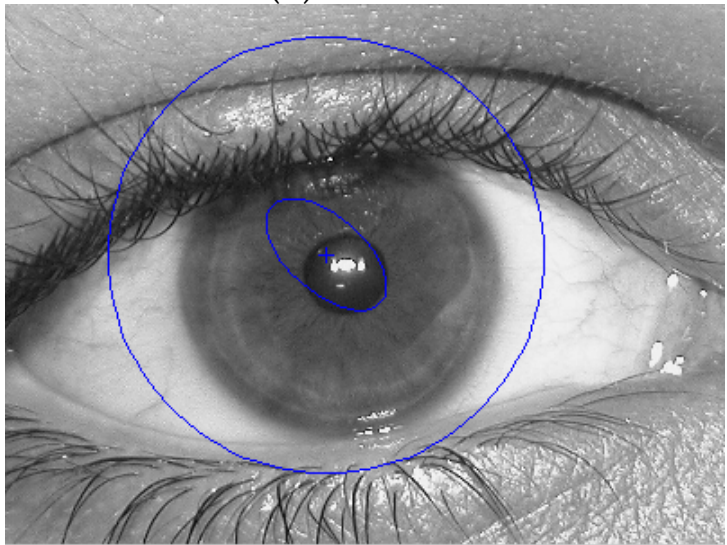

(d) Score: 5

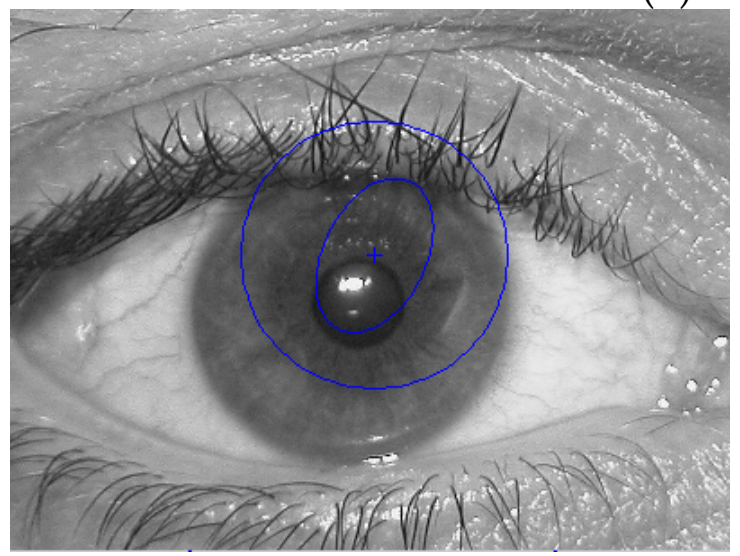

(e) Score: 0

Fig: 4.4: Segmentation Results for UB Iris Database 


\section{Chapter 5}

\section{Towards Future Research}

Although there are iris recognition systems that can operate successfully at $0 \%$ false accept and false reject rates under ideal conditions, there is ample scope for further research in this field. Some of the challenges that are yet to be overcome are enumerated in this chapter.

\section{$5.1 \quad$ Non-Ideal Imaging}

It has been explained in section 1.1.3 what we mean by "non-ideal" images. Our proposed robust pre-processing algorithm takes care of specular reflections, lighting variations, varying angle of acquisition and pupil contraction/dilation. However, a few other factors need more attention. A few research ideas and some preliminary results are explained subsequently.

\subsubsection{Defocus/Motion Blur}

One of the factors contributing to the non-ideality of iris images is the noise introduces because of blurring. There can be various sources that could lead to 
blurred images such as out-of-focus blur, blur due to eye movement or due to contact lenses.

Blurriness could introduce various complications in registration, which in turn could lead to degraded recognition performance. Hence it could prove to be very useful if there was a module in the preprocessing algorithm, which would deblur or enhance the iris images.

This however, is easier said than done. Deblurring is a very challenging problem because of the unknown and probably varying Point Spread Function, which is very difficult to find out or estimate. Also there is an additional risk of producing artifacts due to amplified noise.

\subsubsection{Eyelid/Eyelash Detection}

Our algorithm inherently takes into account the occlusions due to eyelids, eyelashes while doing the segmentation. Most current recognition systems mask the eyelids before calculating the match scores. However, there is scope for future research in this area. The possibility of using information from more than one templates to "fill-in" the areas occluded by eyelids/eyelashes can be explored. This will largely help in reducing the intra-class variability.

\subsubsection{Super-resolution}

The possibility of relaxing the condition on the resolution of the acquired iris images can also be researched further. From this point of view, we studied the performance of Daugman 's algorithm using the CASIA dataset at varying resolutions. The ROC Curve of FAR Vs FRR obtained is as shown in Fig 5.1 


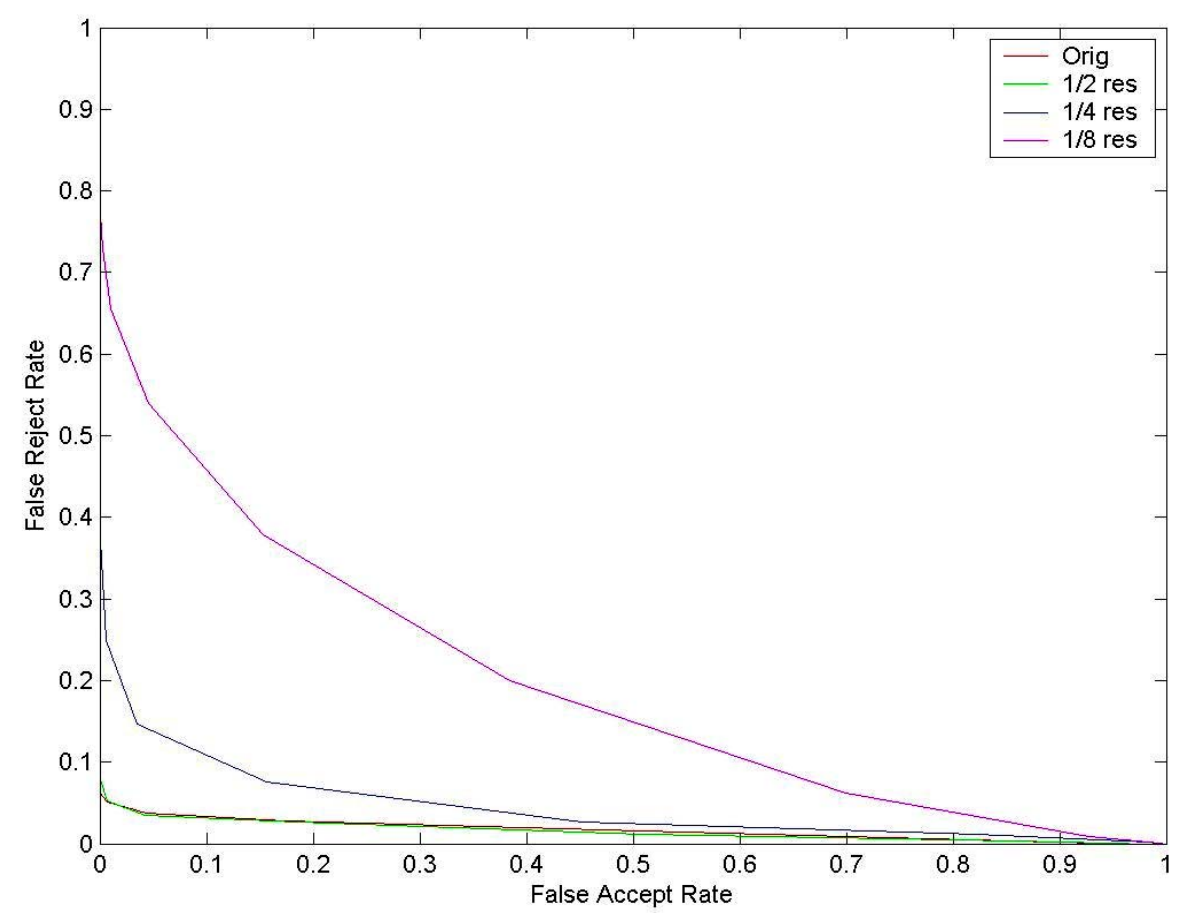

Fig 5.1: ROC Curves for CASIA Dataset using Daugman' s Algorithm

We came across an interesting observation. As can be seen in above plot, the performance curves for original images $\left(320^{*} 280\right)$ and the images having half their resolution overlap. This means both the resolutions give similar performance. We can find the critical resolution required for getting a desirable performance; like $160^{\star} 140$ (half the original resolution) in the above case, which corresponds to an iris pixel width of around 100 pixels. Thus we can relax the conditions on the image capture and use the lower resolution images to make a better quality higher resolution image.

Fig 5.2 shows the results of some of our initial experiments regarding superresolution. 


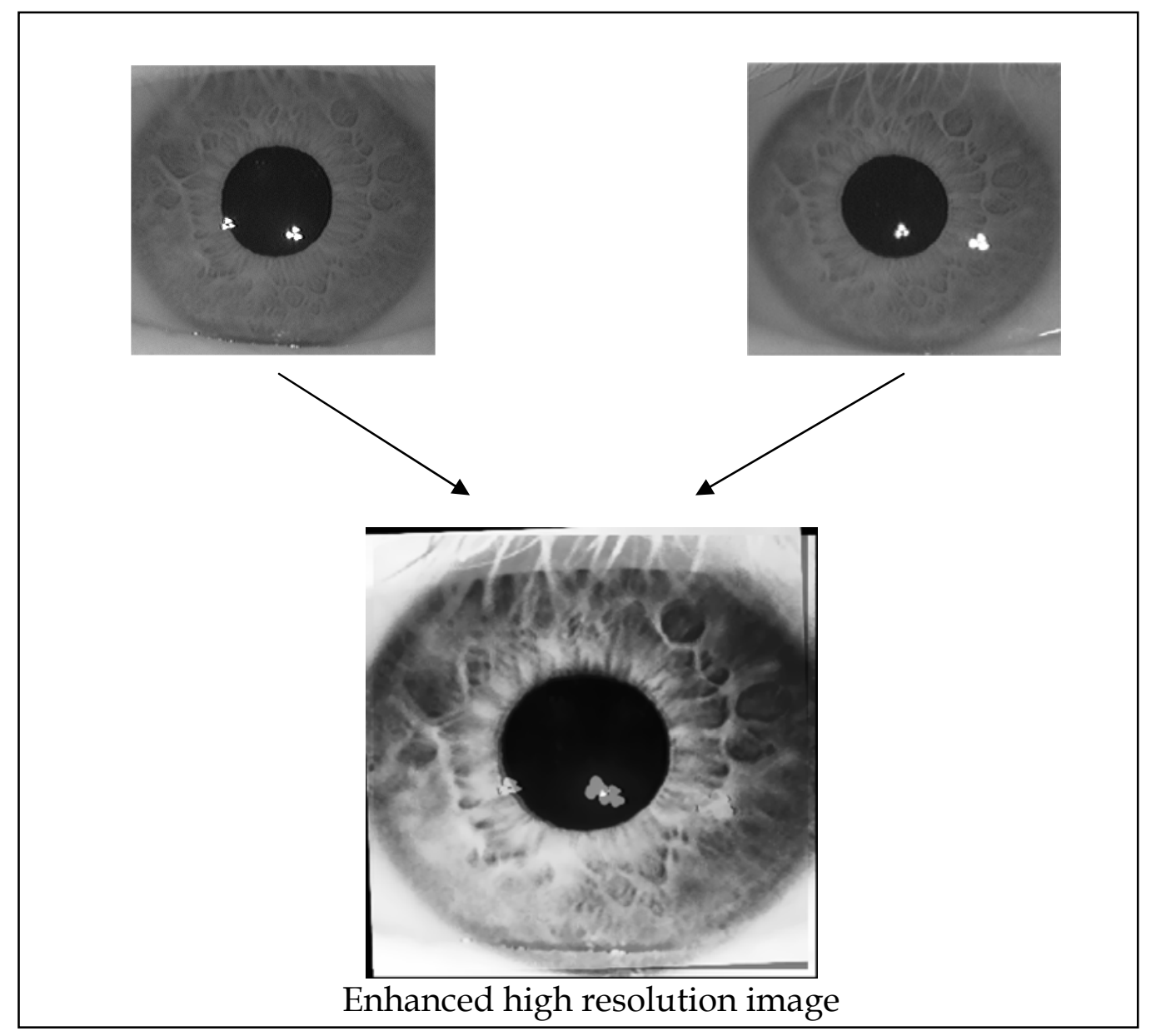

Fig 5.2: Iris Enhancement and super-resolution

This can be very useful in applications where we need to capture the iris image at a distance like in surveillance applications.

\subsection{Iris Classification}

There have been no classification schemes for iris images like those available for fingerprints. Though iris images appear chaotic and random, they do possess a global structure that can be perceived. Most commercial iris recognition systems can perform 1-to-N matches within a couple of seconds. Thus, iris classification may not be of much commercial interest right now, but it is very important from an academic point of view. This will provide more insight into the texture of the 
iris and its individuality. We can explore the possibility of mathematically modeling the different classes.

For iris classification we could use Rayid 's Model of classifying iris texture:

Developed by Denny Ray Johnson, it is a method of interpreting the markings in irises to infer characteristics, patterns, behavioral traits, attitudes and challenges. According to this, iris patterns can be classified into four different classes:

\section{* Stream Iris}

The structure of the Iris is determined by the arrangement of the fibers radiating from the center of the iris (or pupil). Fig 5.3 shows illustrations of stream iris patterns. In the images shown in (a), we notice that they are uniform and reasonably direct or parallel. All irises contain these fibers although in some irises (typically very dark irises) they can be a challenge to discern. The regular arrangement of these fibers is the key to the Stream Iris structure.

Fig 5.3 (b) illustrates the stream iris fiber arrangement. The fibers are roughly parallel, evenly arranged and vary only in density (closeness to each other) and intensity (brightness).
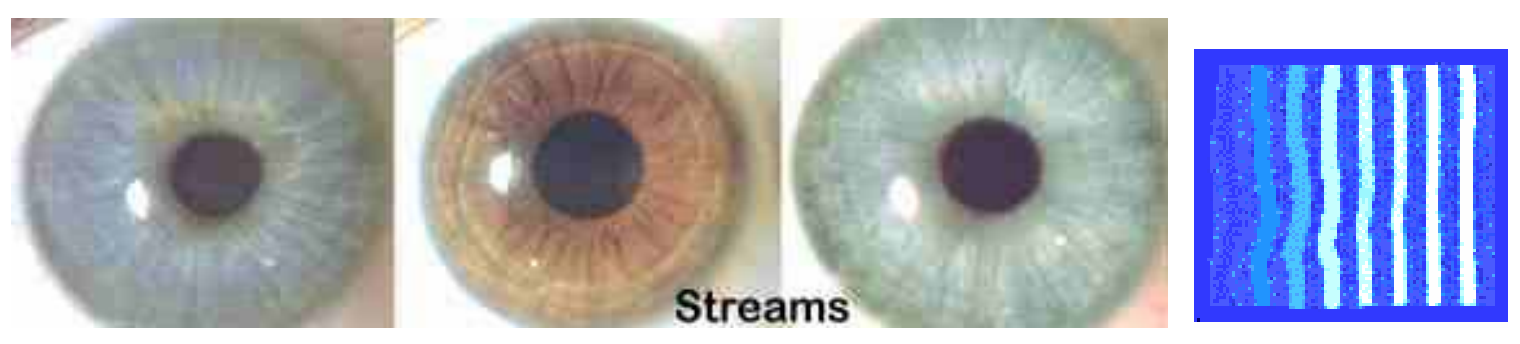

(a) Examples of Stream Iris

(b) Stream fibers

Fig 5.3: Stream Iris 


\section{Flower Iris}

In a Flower Iris the fibers radiating from the center are distorted (in one or more places) to produce the effect of petals (hence the name Flower). In the images shown in Fig 5.4 (a), it can be seen that they are neither regular nor uniform. A flower iris may have only one significant petal with the remainder of the iris looking like a stream. The irregular arrangement of these fibers is the key to the Flower Iris structure.

Fig 5.4 (c) illustrates the flower iris fiber arrangement. The fibers form rounded openings in the iris - these vary in size, density and intensity and are likely to be more apparent in the left eye. This is usually much easier to identify than a stream iris.

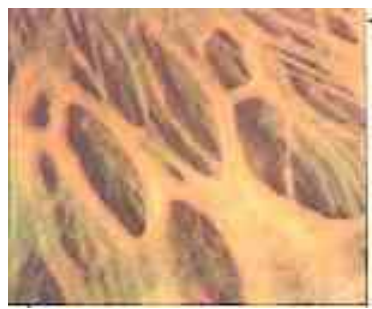

(a) Flower texture

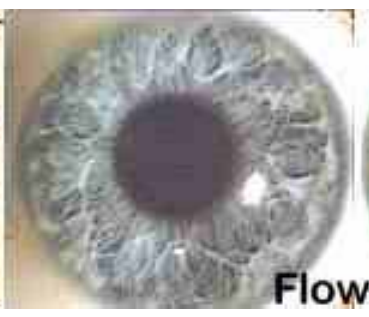

(b) Flower Iris Example

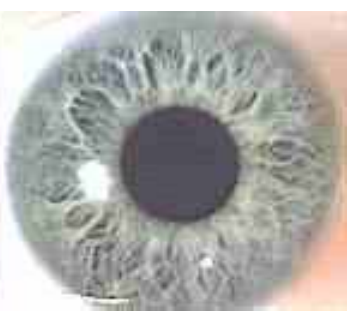

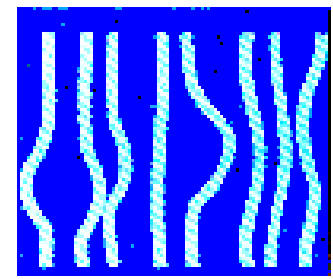

(c) Flower Fibers

Fig 5.4: Flower Iris

\section{Jewel Iris}

The jewel iris can be recognized by the presence of pigmentation or colored dots on top of the fibers. These dots (or jewels) can vary in color from light orange 
through black. They can also vary in size from tiny (invisible to the naked eye) to quite large. The presence of these dots is the key to the Jewel Iris structure.

The image 5.5 (c) illustrates the Jewel iris fiber and pigmentation arrangement. The pigmentation is superimposed above the fibers. This is usually much easier to identify than a stream iris. If an iris looks to be $90 \%$ stream and $10 \%$ jewel it would best to treat it as a jewel.

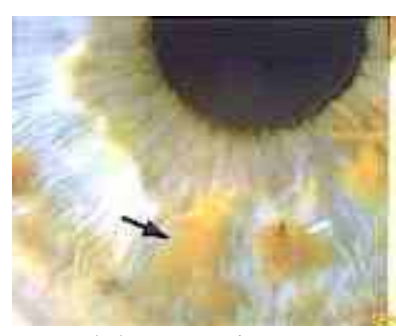

(a) Jewel Iris (b) Examples of jewel irises

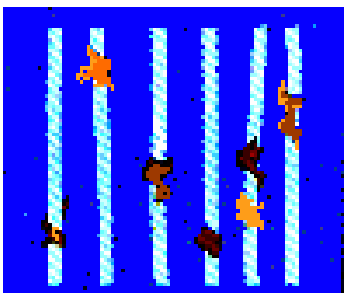

(c) Jewel Fibers

Fig 5.5 Jewel Iris

\section{Shaker Iris}

The Shaker Iris is identified by the presence of both flower-like petals in the fiber arrangement and pigmented dots or jewels. The presence of even one jewel in an otherwise Flower iris is sufficient to cause the occupant to exhibit Shaker characteristics.

The distribution and density of flowers and jewels can vary substantially between the two eyes. The presence of both Flower and Jewel characteristics is the key to the Shaker Iris structure. The image in Fig 5.6 (c) illustrates the Shaker iris fiber arrangement. The fibers form rounded openings in the iris -just as in the 
flower iris. The fibers are overlaid by pigmented dots or jewels as in the jewel iris.

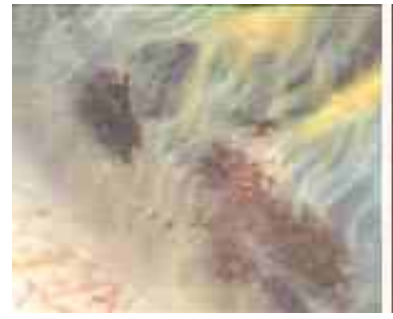

(a) Shaker Pattern

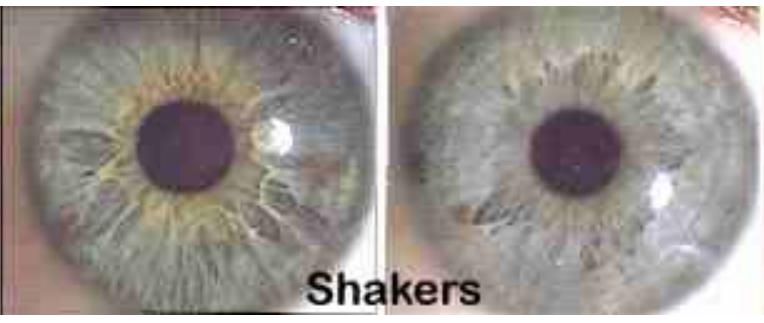

(b) Examples of Shaker Iris
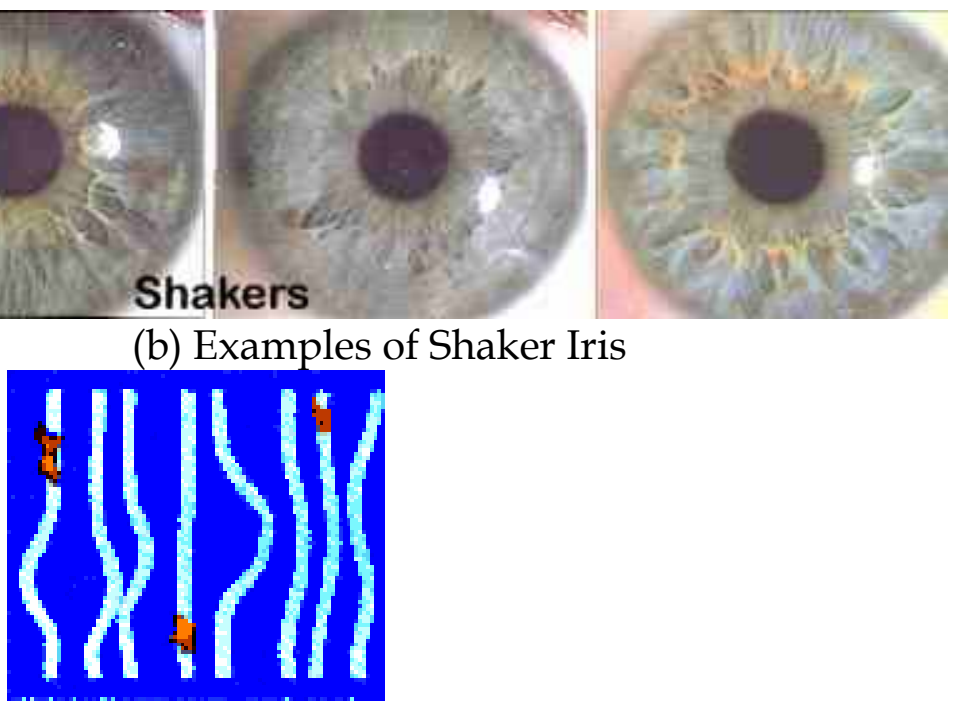

(c) Shaker Fibers

Fig 5.6 Shaker Iris

It is clear from the above classification that even though every iris is unique, there does exist some kind of a global structure. For this study, a database with colored and very high-resolution iris images needs to be collected. Then, after analyzing the iris texture in detail, using available texture analysis techniques, the texture "threads" can be mathematically modeled. Also the possibility of using available pattern classification methods to classify them after modeling can be explored.

\subsection{Using Multiple Templates}

In general more than one template are captured at the time of enrollment. At the time of testing, matching distances are found out with all the templates and the minimum distance is taken as the final value. 
According to our hypothesis, fusing more than one template could help in reducing the intra-class variability. It could turn out to be especially important in cases where one of the templates have been corrupted by noise. It would definitely provide more information than using a single template.

The fusion of templates could be carried out at various levels:

* Feature Level: Fusion at the feature level involves the integration of feature sets corresponding to multiple modalities (here, multiple instances of the same modality). Since the feature set contains richer information about the raw biometric data than the match score or the final decision, integration at this level is expected to provide better recognition results.

- Match Score Level: Here match scores output by multiple matchers are combined. Here, match score using all templates can be found and the fused score can be given out as the final match score. Integration at the matching score level is generally preferred due to the ease in accessing and combining matching scores.

This is also another area that could merit further research. 


\section{Chapter 6}

\section{Conclusion}

Iris as a biometric is gaining a lot of importance in today's world. It has been demonstrated as being a highly accurate and non-invasive biometric. While there exist iris recognition systems that give $0 \%$ false accept and false reject rates under ideal conditions, very few of them consider the factors that come into play in the real world. The goal of this thesis was to develop a pre-processing module, which will make an allowance for such factors leading to "non-ideality" in the captured iris images.

A unique robust segmentation algorithm was designed. Various factors were taken into account here- specular reflections, eyelid/ eyelash occlusions, lighting variations and pupil contraction/dilation. This algorithm is very simple to use because it does not require extensive tuning of parameters as in most previously developed techniques. The only parameters to be assigned here are the minimum pixel count of the pupil boundary, the initial threshold value for binarization and the upper limit on the ratio of the limbus radius to pupil radius. The most important aspect of this algorithm is the approximation of the pupil to an ellipse. Our algorithm thus allows for non-circular shaped pupils. 
The most important contribution of this thesis is compensation of the iris images for capture angle. We came up with a novel angle estimation - compensation algorithm for off-angle images. The highlight of this algorithm is that it automatically detects whether the image is off- angle and estimates its value. We used simple projective transformations for compensation.

It was observed that the results of most current recognition techniques are presented for one particular database. A unique facet of this thesis is that we analyzed the results on four drastically different databases. It was shown that the proposed algorithm works well on all the datasets. We obtained a high success rate of $95.2 \%$ for images from the CASIA Dataset as was expected, since it is a considerably noiseless and ideal database. The other 3 datasets also showed excellent results in spite of being corrupted by various kinds of noise.

Lastly the thesis also dealt with various directions for future research work. 


\section{Bibliography}

[1]. Anil K. Jain, "Biometric recognition: How do I know who you are?", Carnegie Mellon University Distinguished Lecture Series, Nov 2004.

[2]. A. K. Jain, A. Ross and S. Prabhakar, "An Introduction to Biometric Recognition", IEEE Transactions on Circuits and Systems for Video Technology, Special Issue on Image- and Video-Based Biometrics, Vol. 14, No. 1, pp. 4-20, January 2004.

[3]. J. Daugman, "The importance of being random: Statistical principles of iris recognition", Int'1 Conference on Pattern Recognition, vol. 36, no. 2, pp 279-291, 2003.

[4]. John G. Daugman, "High confidence visual recognition of persons by a test of statistical independence", IEEE Trans. on Pattern Analysis and Machine Intelligence, vol. 15, no. 11, pp. 1148-1160, Nov 1993.

[5]. Aaron Lefohn, Brian Budge, Peter Shirley, Richard Caruso, and Erik Reinhard, "An ocularist's approach to human iris synthesis," IEEE Computer Graphics and Applications, vol. 23, no. 6, pp. 70-75, Nov/Dec 2003.

[6]. J. Daugman, "Biometric Personal Identification System Based on Iris Analysis", U.S. Patent, No.5291560, 1994.

[7]. John G. Daugman, "Complete discrete 2-d Gabor transforms by neural networks for image analysis and compression," IEEE Trans. on Acoustics, Speech, and Signal Processing, vol.36, no. 7, pp. 1169-1179, July 1988.

[8]. J. Daugman, “ How Iris Recognition Works", IEEE Conference on Image Processing, 2002. 
[9]. J. Daugman, "Demodulation by complex-valued wavelets for stochastic pattern recognition", Int'l Journal of Wavelets, Multi-resolution and Information Processing, vol. 1, no. 1, pp 1-17,2003.

[10]. L. Flom, A. Safir, "Iris Recognition System", United Sates Patent No. $4641394,1987$.

[11]. Richard P. Wildes, "Iris recognition: An emerging biometric technology", Proceedings of the IEEE, vol. 85, no. 9, pp. 1348-1363, Sept 1997.

[12]. R. Wildes, J. Asmuth, S. Hsu, R. Kolczynski, J. Matey and S. Mcbride, "Automated, Noninvasive Iris Recognition System and Method", U.S. Patent, No.5572596, 1996.

[13]. W.W. Boles and B. Boashash, "A human identification technique using images of the iris and wavelet transform", IEEE Trans. on Signal Processing, vol. 46, no. 4, pp. 1185-1188, Apr 1998.

[14] Li Ma, Yunhong Wang, and Tieniu Tan, "Iris recognition using circular symmmetric filters", Proc. of 16th Intl. Conf. on Pattern Recognition, vol. 2, pp. 805-808, 2000.

[15]. Yong Zhu, Tieniu Tan, Yunhong Wang. "Biometric Personal Identification Based on Iris Patterns," ICPR, vol. 02, p. 2801, 2000.

[16] Li Ma, Tieniu Tan, and Yunhong Wang, "Efficient iris recognition by characterizing key local variations", IEEE Trans. on Image Processing, vol. 13, no. 6, pp. 739-750, June 2004.

[17]. L. Ma, T. Tan, D. Zhang, Y. Wang, "Local Intensity Variation Analysis for Iris Recognition", Pattern Recognition, Vol.37, No.6, pp. 1287-1298, 2004.

[18]. Jiali Cui, Yunhong Wang, Tieniu Tan, Li Ma, Zhenan Sun, "An Iris Recognition Algorithm Using Local Extreme Points", Lecture Notes in Computer Science, Volume 3072, Jan 2004, pp 442 - 449.

[19] Li Ma, Yunhong Wang, and Tieniu Tan, "Personal identification based on iris texture analysis", IEEE Trans. on Pattern Analysis and Machine Intelligence, vol. 25, no. 12, pp. 1519-1533, Dec 2003. 
[20]. Kwanghyuk Bae, Seungin Noh, and Jaihei Kim, "Iris feature extraction using independent component analysis", Proc. 4th Intl. Conf. on Audio and Video Based Biometric Person Authentication (AVBPA 2003), pp. 838-844, 2003.

[21]. Ya-Ping Huang, Si-Wei Luo, En-Yi Chen, “An Efficient Iris Recognition System", Proceedings of the First International Conference on Machine Learning and Cybernetics, pp. 450- 454 vol.1, November 2002.

[22]. Christel- loïc Tisse, Lionel Torres, Michel Robert, "Person Identification Technique Using Human Iris Recognition", Proceedings of the 15th International Conference on Vision Interface, pp. 294-299, 2002.

[23]. L. Masek, Recognition of Human Iris Patterns for Biometric Identification, M.S. Dissertation, The University if Western Australia, 2003, http://www.csse.uwa.edu.au/ pk/studentprojects/libor/

[24]. Krichen, E., Mellakh, M. A., Garcia-Salicetti, S., and Dorizzi, B, "Iris Identification Using Wavelet Packets", In Proceedings of the 17th international Conference on Pattern Recognition, pp. 335-338 vol. 4, August 2004.

[25]. Bolle, R. M., Pankanti, S., Connell, J. H., and Ratha, N. K, "Iris Individuality: A Partial Iris Model", In Proceedings of the 17th international Conference on Pattern Recognition, pp. 927-930, Volume 2, August 2004.

[26]. V. Dorairaj and N. Schmid, and G. Fahmy, Performance Evaluation of Iris Based Recognition System Implementing PCA and ICA Techniques, in Proc. of the SPIE 2005 Symp. on Defense and Security, Conf. 5779:Biometric Technology for Human Identification II, March 28-29, Orlando, FL

[27]. W. Kong and D. Zhang, "Accurate iris segmentation based on novel reflection and eyelash detection model", Proceedings of ISIMVSP, pages 263-266, 2001.

[28]. Jiali Cui, Li Ma, Yunhong Wang, Tieniu Tan, Zhenan Sun, "A Fast and Robust Iris Localization Method based on Texture Segmentation", SPIE, Defense and Security Symposium, Vol. 5404, p. 401-408, 2004.

[29]. Park, Y., Yun, H., Song, M., and Kim, J, " A Fast Circular Edge Detector for the Iris Region Segmentation", In Proceedings of the First IEEE international Workshop on Biologically Motivated Computer Vision, pp. 417 - 423, 2000. 
[30]. Yuanning Liu, Senmiao Yuan, Xiaodong Zhu, Qingliang Cui, "A Practical Iris Acquisition System and A Fast Edges Locating Algorithm in Iris Recognition", Instrumentation and Measurement Technology Conference, pp. 166- 168 vol. 1, May 2003.

[31]. Asheer K. Bachoo, Jules Tapamo, "A segmentation method to improve irisbased person identification", IEEE Africon Conference, 2004.

[32]. R. Gonzalez and R. Woods, "Digital Image Processing", Addison-Wesley Publishing Company, 1992.

[33]. J. Canny, "A Computational Approach to Edge Detection", IEEE Transactions on Pattern Analysis and Machine Intelligence, pp. 679-698, Vol 9, No. 6, Nov 1986.

[34]. A. Fitzgibbon, M. Pilu, and R. B. Fisher, "Direct Least Square Fitting of Ellipses", IEEE Trans. on Pattern Analysis and Machine Intelligence, 21(5): 476480, May 1999.

[35]. Li, X, "Low Bit Rate Image Coding in the Scale Space", Proceedings of the Data Compression Conference, 2002.

[36]. Proença, Hugo and Alexandre, Luís A.,"UBIRIS: A noisy iris image database", Proceedings Of International Conference on Image Analysis and Processing, pp. 970-977 vol. 1, 2005. 